\title{
The indecomposable symplectic and quadratic modules of the Klein-four group
}

\author{
Lars Pforte*, John Murray \\ Department of Mathematics, National University of Ireland, Maynooth, \\ Co. Kildare, Ireland
}

\section{A R T I C L E I N F O}

Article history:

Received 13 July 2017

Available online 7 March 2018

Communicated by Martin Liebeck

\section{Keywords:}

Symplectic and quadratic forms

Indecomposable symplectic and

quadratic modules

Klein-four group

\section{A B S T R A C T}

We determine all indecomposable symplectic modules for the Klein-four group $K_{4}$ over a perfect field of characteristic 2 and classify the symplectic forms up to isometry. We also determine all $K_{4}$-invariant quadratic forms which polarize to a given symplectic form and classify such quadratic forms up to isometry.

() 2018 Elsevier Inc. All rights reserved.

\section{Introduction}

Each linear representation of a finite group $G$ over a field $k$ is a group homomorphism $G \rightarrow \mathrm{GL}(V)$, where $V$ is a finite dimensional $k$-vector space. The isomorphism classes of faithful representations of $G$ correspond to the conjugacy classes of subgroups of general linear groups which are isomorphic to $G$. Likewise a symplectic representation is a homomorphism $G \rightarrow \operatorname{Sp}(V)$ into a symplectic group and an orthogonal representation is a homomorphism $G \rightarrow \mathrm{O}(V)$ into an orthogonal group. The isometry classes of faithful symplectic and orthogonal representations of $G$ then correspond, respectively, to the

\footnotetext{
* Corresponding author.

E-mail addresses: lars.pforte@mu.ie (L. Pforte), john.murray@mu.ie (J. Murray).
} 
conjugacy classes of subgroups of symplectic and orthogonal groups which are isomorphic to $G$. The conjugacy classes of symplectic and orthogonal groups were enumerated by G.E. Wall in [6]. This is essentially equivalent to a classification of the symplectic and orthogonal representations of cyclic groups.

Now let $k G$ be the group algebra of $G$ over $k$. A symplectic $k G$-module is a pair $(M, B)$, where $M$ is a $k G$-module and $B$ is a $G$-invariant symplectic form on $M$. We say that $(M, B)$ is indecomposable if $B$ is degenerate on each proper submodule of $M$. One example of a symplectic $k G$-module can be constructed as follows. Let $M$ be any $k G$-module, with dual $k G$-module $M^{*}=\operatorname{Hom}_{k}(M, k)$. The evaluation $(f, m) \mapsto f(m)$, for $f \in M^{*}$ and $m \in M$, then defines a $G$-invariant quadratic form on $M^{*} \oplus M$. Its polarization is the $G$-invariant symmetric form $P\left((f, m),\left(f^{\prime}, m^{\prime}\right)\right):=f\left(m^{\prime}\right)+f^{\prime}(m)$, for $f, f^{\prime} \in M^{*}$ and $m, m^{\prime} \in M$. This is symplectic in characteristic two. We call $\left(M^{*} \oplus M, P\right)$ the paired module.

From now on, unless otherwise specified, $G$ is the Klein-four group and $k$ is a perfect field of characteristic 2. In this paper we determine the isometry classes of indecomposable symplectic $k G$-modules. For each such module $(M, B)$ we also determine the isometry classes of $G$-invariant quadratic forms on $M$ which polarize to $B$.

As usual $k^{\times}$is the multiplicative group of $k$ and $\mathcal{P}(k)$ is a set of representatives for the cosets of $\left\{\lambda^{2}+\lambda \mid \lambda \in k\right\}$ in the additive group of $k$. Hence $|\mathcal{P}(k)|=2$, if $k$ is finite and $|\mathcal{P}(k)|=1$, if $k$ is algebraically closed. We use $\operatorname{sp}\left(v_{1}, \ldots, v_{n}\right)$ to denote the $k$-span of vectors $v_{1}, \ldots, v_{n}$ in a $k$-vector space and $\lfloor r\rfloor$ to denote the largest integer less than or equal to $r \in \mathbb{R}$. Other notation will be developed as needed. All our modules are left modules.

Recall that $k G$ has tame representation type. In particular there are only finitely many indecomposable $k G$-modules of any given dimension. In Section 4 we review Conlon's classification [1] of these modules. In addition to the trivial module $A_{0}=B_{0}=k_{G}$ and the regular module $D=k G$, the families are

$$
A_{n}, B_{n}, C_{n}(\pi) \text { and } C_{n}(\infty)
$$

where $n$ runs over all positive integers and $\pi$ runs over all irreducible $k$-polynomials. The $k G$-modules which give rise to indecomposable symplectic $k G$-modules are:

$$
\left(k_{G}\right)^{2}, k G,(k G)^{2}, A_{n} \oplus B_{n}, C_{n}(\pi), C_{n}(\pi)^{2}, C_{n}(\infty) \quad \text { and } \quad C_{n}(\infty)^{2}
$$

Now up to scaling $k_{G}$ has a unique symmetric bilinear form, and this form is clearly not symplectic. Moreover any statement about symplectic forms on $k_{G} \oplus k_{G}$ is just a statement about symplectic vector spaces. By [4, Theorem 2.10] if $(k \oplus k, b)$ is a symplectic vector space, then $b$ is a non-zero multiple of the paired form. So $(k \oplus k, b)$ is a hyperbolic plane. The quadratic forms on $k \oplus k$ which polarize to $b$ are parametrized by $d \in k$. Moreover, as $d$ ranges over $\mathcal{P}(k)$, we get representatives for all isometry classes of quadratic forms which polarize to $b$. See [4, Theorem 12.9] and [4, Proposition 13.14] for full details. 
In [3], Gow and Willems described all group invariant symplectic and quadratic forms on the regular module of a finite group. However they did not discuss the isometry classes of such forms. Here we show that the $G$-invariant symplectic forms on $k G$ are parametrized by the triples $(a, b, c) \in k^{3}$ with $a+b+c \neq 0$. Furthermore those triples with $a+b+c=1_{k}$ represent the isometry classes of forms. Also the $G$-invariant quadratic forms which polarize to a given symplectic form are parametrized by $k$, and all such forms are isometric. The details can be found in Theorem 5.1.

The isometry classes of indecomposable $G$-invariant symplectic forms on $(k G)^{2}$ fall into three families: the paired module, a family parametrized by $k^{\times}$and a family parametrized by the projective line $\mathbf{P}^{1}(k)$. So if $k=\mathbb{F}_{q}$ is the field with $q$ elements, there are $2 q+1$ isometry classes of such modules. The $G$-invariant quadratic forms which polarize to a given symplectic form are parametrized by $k^{2}$, and all such forms are isometric. See Theorems 5.2 and 5.3.

For the modules $A_{n} \oplus B_{n}$, the isometry classes of $G$-invariant symplectic forms are parametrized by the vectors in $k^{2 n}$ which have first non-zero coordinate $1_{k}$. This includes the zero vector, which corresponds to the paired module. So if $k=\mathbb{F}_{q}$, there are $1+\left(q^{2 n}-1\right) /(q-1)$ isometry classes. Furthermore there is a $G$-invariant quadratic form which polarizes to a symplectic form in the isometry class of $\left(\lambda_{1}, \ldots, \lambda_{2 n}\right) \in k^{2 n}$ if and only if $\lambda_{2 i}=\lambda_{2 i+1}$, for $i=1, \ldots n-1$. In the case of the paired module there is one isometry class of $G$-invariant quadratic forms. Otherwise the isometry classes of quadratic forms are parametrized by $\mathcal{P}(k)$. For full details, see Theorems 6.4 and 6.7.

The results on symplectic and quadratic forms on $C_{n}(\pi)$ are developed in Section 7.1. Set $K=k[x] /(\pi)$, an extension field of $k$ of degree $m=\operatorname{deg}(\pi)$. Then the isometry classes of $G$-invariant symplectic forms on $C_{n}(\pi)$ are parametrized by $K^{\lfloor n / 2\rfloor}$. So if $k=\mathbb{F}_{q}$ is finite, there are $q^{m\lfloor n / 2\rfloor}$ isometry classes of symplectic forms. For a given $\pi$ and $n \geq 1$, there is at most one isometry class of symplectic forms for which there exist corresponding $G$-invariant quadratic forms. For each symplectic form in this class, the isometry classes of corresponding quadratic forms are parametrized by $\mathcal{P}(k)$. See Theorems 7.5 and 7.7.

We consider the modules $C_{n}(\pi)^{2}$ in Section 7.2. The isometry classes of indecomposable $G$-invariant symplectic forms on $C_{n}(\pi)^{2}$ are parametrized by quadruples $\left(v, w, f_{1}, f_{2}\right)$, where $1 \leq v<w \leq n+1$ with $w-v$ odd and $f_{1}, f_{2} \in K[x]$. Moreover if $w=v+1$, then $f_{1}=0$. Otherwise $\operatorname{deg}\left(f_{1}\right) \leq(w-v-3) / 2$. Also if $w \geq n$, then $f_{2}=1$. Otherwise $\operatorname{deg}\left(f_{2}\right) \leq(n-w-1) / 2$ and $f_{2}(0) \neq 0$. The paired module is given by the quadruple $(n, n+1,0,1)$. In this case there is one isometry class of $G$-invariant quadratic forms. Otherwise corresponding $G$-invariant quadratic forms exist depending on $n+v$, $\pi$ and $f_{1}$. Either $n+v$ is even, $\pi \in\{x, x+1\}$ and $f_{1}=1$ or $n+v$ is odd, $\pi \notin\{x, x+1\}$ and there is one possibility for $f_{1}$. In both cases the isometry classes of quadratic forms are parametrized by $\mathcal{P}(k)$. See Theorems 7.8 and 7.10 .

Finally $C_{n}(\infty)$ is the module $C_{n}(x)$, where the role of two generators of $G$ has been interchanged. Consequently the $G$-invariant symplectic and quadratic forms for $C_{n}(\infty)$ and $C_{n}(\infty) \oplus C_{n}(\infty)$ are the same as for $C_{n}(x)$ and $C_{n}(x) \oplus C_{n}(x)$, respectively. Full details are given in Theorems 8.1, 8.3, 8.4 and 8.6. 
We recall basic results and define notation for matrices in Section 3. These are used throughout the remainder of the paper. Section 3.2 outlines the algebraic background needed to understand the modules $C_{n}(\pi)$ and $C_{n}(\infty)$.

\section{Forms and modules}

The theory of symmetric, symplectic and quadratic forms is highly dependent on whether the characteristic is odd or even. In this section we give full definitions and briefly overview the theory of forms in both cases.

\subsection{Bilinear and quadratic forms}

Let $M$ be a finite dimensional vector space over a field $k$, with dual space $M^{*}$ and endomorphism ring $\operatorname{End}_{k}(M)$. Fix an ordered basis $\mathcal{M}=\left(m_{1}, \ldots, m_{n}\right)$ of $M$ and let $\mathcal{M}^{T}=\left(m_{1}^{T}, \ldots, m_{n}^{T}\right)$ be the dual basis of $M^{*}$. So $m_{i}^{T} m_{j}=\delta_{i j}$, for all $i, j$. This extends to a $k$-isomorphism $T: M \rightarrow M^{*}, m \mapsto m^{T}$, for all $m \in M$. Note that $m^{T} m^{\prime}=\left(m^{\prime}\right)^{T} m$, for all $m, m^{\prime} \in M$.

A bilinear form on $M$ is a map $B: M \times M \rightarrow k$ which is linear in both variables. Given $B$, there is a unique $\beta \in \operatorname{End}_{k}(M)$ such that $B\left(m, m^{\prime}\right)=m^{T} \beta m^{\prime}$. This is clear as $B$ and $\beta$ are uniquely determined by the values $B\left(m_{i}, m_{j}\right)$ and $m_{i}^{T} \beta m_{j}$, for all $i, j=$ $1, \ldots, n$, respectively. In this sense we can identify $B$ and $\beta$. Also we can identify $B$ and the Gram matrix $\left(m_{i}^{T} \beta m_{j}\right)_{1 \leq i, j \leq n}$. Note that this identification depends on the choice of $\mathcal{M}$. We say that $B$ is non-degenerate if $\beta$, or equivalently $\left(m_{i}^{T} \beta m_{j}\right)_{1 \leq i, j \leq n}$ is invertible.

Next define $B^{T}\left(m, m^{\prime}\right):=B\left(m^{\prime}, m\right)$, for all $m, m^{\prime} \in M$. Then $B \rightarrow B^{T}$ is an involuntary $k$-algebra anti-automorphism of $\operatorname{End}_{k}(M)$. Note that the Gram matrix of $B^{T}$ is the transpose of the Gram matrix of $B$. Also $m^{T} B^{T} m^{\prime}=\left(m^{\prime}\right)^{T} B m=(B m)^{T} m^{\prime}$, and thus $(B m)^{T}=m^{T} B^{T}$, for all $m \in M$.

We say that $B$ is symmetric if $B^{T}=B$, and skew-symmetric if $B^{T}=-B$. These are mutually exclusive properties if $\operatorname{char}(k) \neq 2$, but they are equivalent if $\operatorname{char}(k)=2$. We say that $B$ is alternating if $B(m, m)=0$, for all $m \in M$. It is easy to check that each alternating form is skew-symmetric. Also if $\operatorname{char}(k) \neq 2$, each skew-symmetric form is alternating. However if $\operatorname{char}(k)=2$, a form $B$ is alternating if and only if it is symmetric and hollow (meaning $B\left(m_{i}, m_{i}\right)=0$, for $i=1, \ldots, n$ ).

We say that $B$ is a symplectic form if it is both alternating and non-degenerate. In this case we call $(M, B)$ a symplectic space. Then $M$ has an ordered basis which represents $B$ as a diagonal sum of $2 \times 2$ block matrices $\left(\begin{array}{rr}0 & 1 \\ -1 & 0\end{array}\right)$. In particular $\operatorname{dim}(M)$ is even. Conversely each even dimensional space has one symplectic form, up to isometry.

Next let $B$ be non-degenerate and let $\alpha \in \operatorname{End}_{k}(M)$. We define $B_{\alpha}\left(m, m^{\prime}\right):=$ $B\left(m, \alpha m^{\prime}\right)$, for all $m, m^{\prime} \in M$. Then $B_{\alpha}$ is a bilinear form on $M$ and $B_{\alpha}=B \alpha$. In particular $B_{\alpha}$ is non-degenerate if and only if $\alpha$ is invertible. Moreover $\left\{B_{\alpha} \mid \alpha \in\right.$ $\left.\operatorname{End}_{k}(M)\right\}$ uniquely describes all bilinear forms on $M$. For instance $B^{T}=B_{\sigma}$, where $\sigma=B^{-1} B^{T}$. Also there is a unique $\alpha^{o} \in \operatorname{End}_{k}(M)$ so that $B\left(\alpha m, m^{\prime}\right)=B\left(m, \alpha^{o} m^{\prime}\right)$, for 
all $m, m^{\prime} \in M$. We call the map ${ }^{o}$ the adjoint of $B$. As $(\alpha m)^{T}=m^{T} \alpha^{T}$, for all $m \in M$, we have $\alpha^{o}=B^{-1} \alpha^{T} B$. So ${ }^{o}$ is a $k$-algebra anti-automorphism of $\operatorname{End}_{k}(M)$. Also note that $\sigma^{o}=\sigma^{-1}$.

Next, a quadratic form on $M$ is a map $q: M \rightarrow k$ such that $q(\lambda m)=\lambda^{2} q(m)$, for all $\lambda \in k, m \in M$, and $q\left(m+m^{\prime}\right)=q(m)+q\left(m^{\prime}\right)+P_{q}\left(m, m^{\prime}\right)$, for all $m, m^{\prime} \in M$, where $P_{q}$ is a bilinear form, called the polarization of $q$. We note that $P_{q}$ is symmetric. If $P_{q}$ is non-degenerate, we say that $q$ is non-degenerate and call $(M, q)$ a quadratic space.

Now suppose that $B$ is a bilinear form. Then the diagonal of $B$ is the quadratic form

$$
\Delta B(m):=B(m, m), \quad \text { for all } m \in M
$$

Notice that $\Delta B$ polarizes to $B+B^{T}$ and $\Delta B=0$ if and only if $B$ is alternating. In particular $\Delta B=\Delta B^{\prime}$, for bilinear forms $B$ and $B^{\prime}$, if and only if $B-B^{\prime}$ is alternating.

Now $q=\frac{1}{2} \Delta P_{q}$ if $\operatorname{char}(k) \neq 2$. However if $\operatorname{char}(k)=2$, this does not make sense and indeed $P_{q}$ is alternating and $q$ cannot be recovered from $P_{q}$. To rectify this situation, we define the upper-triangular part of $B$ as the bilinear form

$$
\nabla B\left(m_{i}, m_{j}\right):= \begin{cases}B\left(m_{i}, m_{j}\right), & \text { if } i<j . \\ 0, & \text { if } i \geq j .\end{cases}
$$

Of course, $\nabla B$ depends on the choice $\mathcal{M}$ of an ordered basis. Also define $D_{q}\left(m_{i}, m_{j}\right)=$ $q\left(m_{i}\right) \delta_{i j}$, for all $i, j$. Then $D_{q}$ defines a bilinear form which has a diagonal matrix relative to $\mathcal{M}$. Moreover $q=\Delta\left(D_{q}+\nabla P_{q}\right)$, or more concretely

$$
q\left(\sum_{i=1}^{n} \lambda_{i} m_{i}\right)=\sum_{i=1}^{n} \lambda_{i}^{2} q\left(m_{i}\right)+\sum_{1 \leq i<j \leq n} \lambda_{i} \lambda_{j} P_{q}\left(m_{i}, m_{j}\right), \quad \text { for all } \lambda_{1}, \ldots, \lambda_{n} \in k \text {. }
$$

Conversely, given an alternating form $B$, it is clear that

$$
\{\Delta(D+\nabla B) \mid D \text { diagonal }\} \text { is the set of all quadratic forms which polarize to } B \text {. }
$$

\subsection{Symplectic and quadratic modules}

Now suppose that $M$ is a $k G$-module and let $\operatorname{End}_{G}(M)$ be the algebra of $k G$-endomorphisms of $M$. Identify $g \in G$ with $m \mapsto g m$ in $\operatorname{End}_{k}(M)$. Then $\operatorname{End}_{G}(M)=$ $\left\{\alpha \in \operatorname{End}_{k}(M) \mid g \alpha=\alpha g\right\}$. We say that a bilinear form $B$ on $M$ is $G$-invariant if $B\left(g m, g m^{\prime}\right)=B\left(m, m^{\prime}\right)$, for all $g \in G$ and $m, m^{\prime} \in M$. Hence $B$ is $G$-invariant if and only if $g^{T} B g=B$, for all $g \in G$.

We say that $M$ is self-dual if $M \cong M^{*}$ as $k G$-modules. Given a $k G$-isomorphism $\phi: M \rightarrow M^{*}$, set $B\left(m, m^{\prime}\right):=\phi(m)\left(m^{\prime}\right)$, for all $m, m^{\prime} \in M$. Then $B$ is a nondegenerate $G$-invariant bilinear form on $M$. Conversely, each non-degenerate $G$-invariant 
bilinear form on $M$ defines a $k G$-isomorphism $M \cong M^{*}$. So $M$ affords a non-degenerate $G$-invariant bilinear form if and only if $M$ is self-dual.

Let $B$ be a non-degenerate $G$-invariant bilinear form on $M$. For $\alpha \in \operatorname{End}_{k}(M)$ recall $B_{\alpha}$ as defined in section 2.1. Then $B_{\alpha}$ is $G$-invariant if and only if $\alpha \in$ $\operatorname{End}_{G}(M)$. Overall the set of $G$-invariant symplectic forms on $M$ is $\left\{B_{\alpha} \mid \alpha \in\right.$ $\operatorname{Aut}_{G}(M)$ and $B \alpha$ is alternating $\}$.

Next suppose that $B$ is a $G$-invariant symplectic form. We then call $(M, B)$ a symplectic $k G$-module. For each submodule $N$ of $M$, set $N^{\perp}:=\{m \in M \mid B(m, n)=0$, for all $n \in N\}$. Then $N^{\perp}$ is a submodule of $M$ such that $M / N^{\perp} \cong N^{*}$. In particular $\operatorname{dim}(M)=\operatorname{dim}(N)+\operatorname{dim}\left(N^{\perp}\right)$. Hence if $B$ restricts to a non-degenerate form on $N$, then $M=N \oplus N^{\perp}$, as $k G$-modules, and $\left(N^{\perp}, B\right)$ is symplectic. We say that $(M, B)$ is indecomposable if it is not a sum of two proper symplectic $k G$-modules. The proposition in [2] (and its proof) shows that:

Lemma 2.1. Let $(M, B)$ be a symplectic $k G$-module and let $M=M_{1} \oplus \cdots \oplus M_{t}$ be a decomposition of $M$ into indecomposable $k G$-modules. Then for each $i \in\{1, \ldots, t\}$ either $M_{i}$ is non-degenerate or there exists $j \neq i$ such that $B: M_{i} \times M_{j} \rightarrow k$ is a non-degenerate pairing. In particular $M_{j} \cong M_{i}^{*}$ and $B$ is non-degenerate on $M_{i} \oplus M_{j}$.

The lemma implies that $(M, B)$ is indecomposable if and only if $M$ is indecomposable, or $M \cong N \oplus N^{*}$, where $N$ is indecomposable and $(N, B)$ is degenerate.

We say that $\alpha:\left(M, B_{1}\right) \rightarrow(M, B)$ is an isometry of symplectic $k G$-modules if $\alpha \in$ $\operatorname{End}_{G}(M)$ and $B_{1}\left(m, m^{\prime}\right)=B\left(\alpha m, \alpha m^{\prime}\right)$, for all $m, m^{\prime} \in M$ (or equivalently if $B_{1}=$ $\left.\alpha^{T} B \alpha\right)$. For example, $m \mapsto \mu m$, for $\mu \in k^{\times}$, defines an isometry $\left(M, \mu^{2} B\right) \cong(M, B)$.

Note that an isometry is necessarily a $k G$-isomorphism. Clearly isometry is an equivalence relation on the $G$-invariant symplectic forms on $M$. We fix a set of representatives $\operatorname{Isom}(M)$ for the corresponding equivalence classes.

Next let $q$ be a quadratic form on $M$. Then for each $g \in G$, the map $m \mapsto q(\mathrm{gm})$, for $m \in M$, is a quadratic form on $M$. Moreover $q(g-)$ polarizes to $g^{T} P_{q} g$. So $q$ and $q(g-)$ polarize to $P_{q}$, if $P_{q}$ is $G$-invariant. We say that $q$ is $G$-invariant if $q(g-)=q$, for all $g \in G$. Now suppose that $q=\Delta Q$, for some bilinear form $Q$. Then $q(g-)=\Delta\left(g^{T} Q g\right)$. As a consequence

$q$ is $G$-invariant if and only if $Q-g^{T} Q g$ is alternating, for each $g \in G$.

Notice that (1) implies that if $P_{q}$ is $G$-invariant, then $q$ is $G$-invariant if and only if $q\left(g m_{i}\right)=q\left(m_{i}\right)$, for $i=1, \ldots, n$ and all $g \in G$.

Let $q^{\prime}$ be another $G$-invariant quadratic form on $M$. We say that $\alpha \in \operatorname{End}_{G}(M)$ is an isometry $(M, q) \rightarrow\left(M, q^{\prime}\right)$ if $q^{\prime}(m)=q(\alpha m)$, for all $m \in M$. Notice that if $\alpha$ exists then it induces an isometry $\left(M, P_{q}\right) \rightarrow\left(M, P_{q^{\prime}}\right)$.

Now suppose that $q$ is $G$-invariant with polarization $B$ and let $\left(M, B^{\prime}\right)$ be isometric to $(M, B)$. Then $B^{\prime}=\beta^{T} B \beta$, for some invertible $\beta \in \operatorname{End}_{G}(M)$. Then the quadratic 
form $\beta^{T} q \beta$ is $G$-invariant and $\beta:(M, q) \rightarrow\left(M, \beta^{T} q \beta\right)$ is an isometry. This discussion shows that once $\operatorname{Isom}(M)$ is fixed, each isometry class of non-degenerate $G$-invariant quadratic forms on $M$ contains a form $q$ such that $P_{q} \in \operatorname{Isom}(M)$. We let $\operatorname{QIsom}(M, B)$ be a full set of representatives for the distinct isometry classes of $G$-invariant quadratic forms on $M$ which polarize to $B$.

\subsection{Pairings between a module and its dual}

Let $k$ be a field of characteristic 2 and let $M$ be an indecomposable $k G$-module. Equivalently $\operatorname{End}_{G}(M)$ is a local ring, in the sense that every element of $\operatorname{End}_{G}(M)$ is either nilpotent or invertible. Let $\mathrm{J}\left(\operatorname{End}_{G}(M)\right)$ be the Jacobson radical of $\operatorname{End}_{G}(M)$. Then $\overline{\operatorname{End}_{G}(M)}:=\operatorname{End}_{G}(M) / \mathrm{J}\left(\operatorname{End}_{G}(M)\right)$ is a division algebra over $k$. In particular $\overline{\operatorname{End}_{G}(M)}$ is a field if $k$ is finite, and $\overline{\operatorname{End}_{G}(M)} \cong k$ if $k$ is algebraically closed.

Suppose that $M \nsubseteq M^{*}$. Then $M^{*} \oplus M$ has no self-dual indecomposable direct summand. So the paired module $\left(M^{*} \oplus M, P\right)$, as defined in the introduction, is indecomposable.

For the rest of this section we assume that $M$ is self-dual. We briefly discuss symplectic forms on $M^{2}$, as we face this situation in Sections 7.2 and 8 .

We identify $M^{2}$ with ordered pairs $\left(\begin{array}{c}m_{1} \\ m_{2}\end{array}\right)$ of elements of $M$. The $k G$-endomorphisms of $M^{2}$ are $\left(\begin{array}{ll}a & b \\ c & d\end{array}\right)\left(\begin{array}{l}m_{1} \\ m_{2}\end{array}\right):=\left(\begin{array}{c}a m_{1}+b m_{2} \\ c m_{1}+d m_{2}\end{array}\right)$, for all $\left(\begin{array}{c}m_{1} \\ m_{2}\end{array}\right) \in M^{2}$, where $a, b, c, d \in \operatorname{End}_{G}(M)$. Likewise the $G$-invariant bilinear forms on $M^{2}$ are

$$
\left(\begin{array}{cc}
A & B \\
C & D
\end{array}\right)\left(\left(\begin{array}{c}
m_{1} \\
m_{2}
\end{array}\right),\left(\begin{array}{c}
m_{3} \\
m_{4}
\end{array}\right)\right):=A\left(m_{1}, m_{3}\right)+B\left(m_{1}, m_{4}\right)+C\left(m_{2}, m_{3}\right)+D\left(m_{2}, m_{4}\right),
$$

for all $\left(\begin{array}{c}m_{1} \\ m_{2}\end{array}\right),\left(\begin{array}{c}m_{3} \\ m_{4}\end{array}\right) \in M^{2}$, where $A, B, C, D$ are $G$-invariant bilinear forms on $M$.

Let $\left(\begin{array}{cc}A & B \\ C & D\end{array}\right)$ be an indecomposable symplectic form on $M^{2}$. As $\left(\begin{array}{cc}A & B \\ C & D\end{array}\right)^{T}=\left(\begin{array}{ll}A^{T} & C^{T} \\ B^{T} & D^{T}\end{array}\right)$, $A$ and $D$ are alternating and $C=B^{T}$. Lemma 2.1 implies that $A$ and $D$ are degenerate and $B$ is non-degenerate.

Lemma 2.2. Suppose that $\overline{\operatorname{End}_{G}(M)}$ is a field and that the adjoint of some (and thus all) non-degenerate $G$-invariant form on $M$ acts trivially on $\overline{\operatorname{End}_{G}(M)}$. Then the indecomposable $G$-invariant symplectic forms on $M^{2}$ are

$$
\left\{\left(\begin{array}{cc}
A & B \\
B^{T} & D
\end{array}\right) \mid \begin{array}{r}
B \text { non-degenerate } G \text {-invariant bilinear form on } M \\
A, D \text { degenerate } G \text {-invariant alternating forms on } M
\end{array}\right\} .
$$

Proof. Let $\mu: \operatorname{End}_{G}(M) \rightarrow \overline{\operatorname{End}_{G}(M)}$ be the projection, with kernel $\mathrm{J}\left(\operatorname{End}_{G}(M)\right)$. The assumption on $\overline{\operatorname{End}_{G}(M)}$ implies that $M$ is indecomposable. So every indecomposable direct summand of $M^{2}$ is the image of an injective $k G$-homomorphism $\phi: M \rightarrow M^{2}$. We can write $\phi(m)=\left(\begin{array}{c}\phi_{1} m \\ \phi_{2} m\end{array}\right)$, for all $m \in M$, where $\phi_{1}, \phi_{2} \in \operatorname{End}_{G}(M)$. Then for $m_{1}, m_{2} \in M$ we have 


$$
\begin{array}{r}
\left(\begin{array}{cc}
A & B \\
B^{T} & D
\end{array}\right)\left(\left(\begin{array}{c}
\phi_{1} m_{1} \\
\phi_{2} m_{1}
\end{array}\right),\left(\begin{array}{c}
\phi_{1} m_{2} \\
\phi_{2} m_{2}
\end{array}\right)\right)=A\left(\phi_{1} m_{1}, \phi_{1} m_{2}\right)+B\left(\phi_{1} m_{1}, \phi_{2} m_{2}\right)+ \\
B^{T}\left(\phi_{2} m_{1}, \phi_{1} m_{2}\right)+D\left(\phi_{2} m_{1}, \phi_{2} m_{2}\right)
\end{array}
$$

Recall from section 2.1 that $A=B_{\alpha}$ and $D=B_{\delta}$, for some $\alpha, \delta \in \mathrm{J}\left(\operatorname{End}_{G}(M)\right)$, and let $\sigma=B^{-1} B^{T}$. Then the right hand side equals $B_{e}\left(m_{1}, m_{2}\right)$, with $e=\phi_{1}^{o} \alpha \phi_{1}+\phi_{1}^{o} \phi_{2}+$ $\phi_{2}^{o} \sigma \phi_{1}+\phi_{2}^{o} \delta \phi_{2}$. So $\mu(e)=\mu\left(\phi_{1}^{o} \phi_{2}+\phi_{2}^{o} \sigma \phi_{1}\right)$, using $\mu\left(\phi_{1}^{o} \alpha \phi_{1}\right)=\mu\left(\phi_{2}^{o} \delta \phi_{2}\right)=0$.

Next the hypothesis on $\overline{\operatorname{End}_{G}(M)}$ implies that $\mu\left(\zeta^{o}\right)=\mu(\zeta)^{o}=\mu(\zeta)$, for all $\zeta \in$ $\operatorname{End}_{G}(M)$. In particular $\mu(\sigma)^{-1}=\mu\left(\sigma^{-1}\right)=\mu\left(\sigma^{o}\right)=\mu(\sigma)$. This forces $\mu(\sigma)=1$, as $\overline{\operatorname{End}_{G}(M)}$ is a field of characteristic 2 . So

$$
\mu(e)=\mu\left(\phi_{1}^{o}\right) \mu\left(\phi_{2}\right)+\mu\left(\phi_{2}^{o}\right) \mu(\sigma) \mu\left(\phi_{1}\right)=0
$$

We deduce that $e \in \mathrm{J}\left(\operatorname{End}_{G}(M)\right)$. So $\left(\begin{array}{cc}A & B \\ B^{T} & D\end{array}\right)$ is degenerate on $\phi M$. In particular we conclude that $\left(M^{2},\left(\begin{array}{rr}A & B \\ B^{T} & D\end{array}\right)\right)$ is indecomposable.

Let $B$ be any non-degenerate $G$-invariant bilinear form on $M$. Recall that $\left(m_{1}, \ldots, m_{n}\right)$ and $\left(\ell_{1}, \ldots, \ell_{n}\right)$ are $B$-dual bases of $M$ if $B\left(\ell_{i}, m_{j}\right)=\delta_{i j}$, for all $i, j$. Then $\left(\ell_{i}, m_{j}\right) \mapsto$ $\left(m_{i}^{T}, m_{j}\right)$, for all $i, j$, extends to an isometry $\left(M^{2},\left(\begin{array}{cc}0 & B \\ B^{T} & 0\end{array}\right)\right) \rightarrow\left(M^{*} \oplus M, P\right)$. So under the hypothesis of Lemma 2.2 , the paired module $\left(M^{*} \oplus M, P\right)$ is indecomposable.

We note that there is a 2-dimensional self-dual indecomposable $\mathbb{F}_{2} C_{3}$-module $M$ whose paired module is decomposable. In this case $\operatorname{End}_{G}(M) \cong \mathbb{F}_{4}$ and the adjoint of a symplectic form on $M$ acts as the Frobenius automorphism $\lambda \mapsto \lambda^{2}$ on $\mathbb{F}_{4}$.

\section{Matrices}

Let $k$ be a perfect field of characteristic 2 . For all $m, n \geq 1$, we let $\operatorname{Mat}_{m \times n}(k)$ be the $k$-space of $m \times n$ matrices with entries in $k$, simplified to $\operatorname{Mat}_{n}(k)$ if $m=n$.

\subsection{Definitions and notation}

Suppose that $M \in \operatorname{Mat}_{n}(k)$. Then the centralizer and transposer of $M$ are

$$
\begin{aligned}
& \mathrm{C}_{\operatorname{Mat}_{n}(k)}(M):=\left\{X \in \operatorname{Mat}_{n}(k) \mid X M=M X\right\}, \\
& \mathrm{T}_{\operatorname{Mat}_{n}(k)}(M):=\left\{X \in \operatorname{Mat}_{n}(k) \mid X M=M^{T} X\right\} .
\end{aligned}
$$

We simplify to $\mathrm{C}(M)$ and $\mathrm{T}(M)$, if $n$ and $k$ are clear from the context. Then $\mathrm{C}(M)$ is a subalgebra of $\operatorname{Mat}_{n}(k)$, and $\mathrm{T}(M)=\mathcal{T} \mathrm{C}(M)$, if $\mathcal{T}$ is an invertible matrix in $\mathrm{T}(M)$. Now $\mathrm{C}\left(U^{-1} M U\right)=U^{-1} \mathrm{C}(M) U$, for all $U \in \mathrm{GL}_{n}(k)$. Moreover

Lemma 3.1. If $U \in \mathrm{GL}_{n}(k)$ then $\mathrm{T}\left(U^{-1} M U\right)=U^{T} \mathrm{~T}(M) U$.

We say that $M$ is alternating if it is hollow (has a zero diagonal) and symmetric. Then a pairing argument gives: 
Lemma 3.2. Let $M \in \operatorname{Mat}_{n}(k)$ be symmetric and let $U \in \mathrm{GL}_{n}(k)$. Then $M$ is hollow if and only if $U^{T} M U$ is hollow. Moreover, for $V \in \operatorname{Mat}_{n}(k)$ we have

$$
\left(V^{T} M V\right)_{i i}=\sum_{j=1}^{n} M_{j j} V_{j i}^{2}, \quad \text { for each } i \in\{1, \ldots, n\} .
$$

We use $E_{s, t}$ to denote a matrix, of unspecified dimensions, which has the single nonzero entry 1 at position $(s, t)$. We simplify to $E_{s}$ for the diagonal matrix $E_{s, s}$. By fiat $E_{s, t}$ is the zero matrix in $\operatorname{Mat}_{m \times n}(k)$, if $s \notin\{1, \ldots, m\}$ or $t \notin\{1, \ldots, n\}$. We use $\operatorname{Diag}_{n}(k)$ to denote the diagonal matrices in $\operatorname{Mat}_{n}(k)$. We write $\operatorname{diag}\left(A_{1}, \ldots, A_{t}\right)$ for a matrix which has the square blocks $A_{1}, \ldots, A_{t}$ positioned successively along its diagonal. We use $\star$ to denote a block of arbitrary entries and · to denote a block with 0 entries in any matrix.

A matrix is Toeplitz if it has constant diagonals. For each integer $s$ we define $T_{s}:=$ $\sum_{i} E_{i, i+s-1}$. So $T_{s}$ is a non-zero Toeplitz matrix in $\operatorname{Mat}_{m, n}(k)$, if $2-m \leq s \leq n$. Moreover $\left(T_{2-m}, \ldots, T_{n}\right)$ is a basis for the space $\mathcal{T}_{m \times n}(k)$ of all $m \times n$ Toeplitz matrices. We use $\mathcal{T}_{n}(k)$ to refer to $\mathcal{T}_{n \times n}(k)$.

A matrix is Hankel if it has constant anti-diagonals. For each integer $s$ we define $H_{s}:=\sum_{i} E_{i, s-i}$. So $H_{s}$ is a non-zero Hankel matrix in $\operatorname{Mat}_{m, n}(k)$, if $2 \leq s \leq m+n$. Moreover $\left(H_{2}, \ldots, H_{m+n}\right)$ is a basis for the space $\mathcal{H}_{m \times n}(k)$ of all $m \times n$ Hankel matrices. We use $\mathcal{H}_{n}(k)$ to refer to $\mathcal{H}_{n \times n}(k)$. Notice that each square Hankel matrix is symmetric.

We call a Hankel matrix monic if its first non-zero row has first non-zero entry 1 , and we use $\mathcal{M H}_{m \times n}(k)$ to denote the set of all monic $m \times n$ Hankel matrices. Now label the anti-diagonal containing $(i, j)$ by $i+j$. Then we use $\mathcal{D} \mathcal{H}_{(n+1) \times n}(k)$ to denote the set of monic $(n+1) \times n$ Hankel matrices which are constant on the anti-diagonals labeled by $2 i-1$ and $2 i$, for all $i=2, \ldots n$. Note that both $\mathcal{M H}_{m \times n}(k)$ and $\mathcal{D} \mathcal{H}_{(n+1) \times n}(k)$ contain the zero matrix.

We write $\widetilde{I}_{n}$ for the $n \times n$ Hankel matrix $H_{n+1}$, simplified to $\widetilde{I}$ if $n$ is understood. We call $\widetilde{I}$ the exchange matrix. So $\widetilde{I}$ has 1 's on its main anti-diagonal. Note that $\widetilde{I}^{2}=I$ and that $H_{s}$ is non-singular if and only if $H_{s}=\widetilde{I}$. Also $\widetilde{I}_{n} T_{s}=H_{n+s}$. So $\widetilde{I}_{n} \cdot \mathcal{T}_{n \times m}(k)=\mathcal{H}_{n \times m}(k)$.

For all $m, n \geq 1$, exchange-transpose is a map $\operatorname{Mat}_{m \times n}(k) \rightarrow \operatorname{Mat}_{n \times m}(k)$ defined by

$$
A^{\widetilde{T}}:=\widetilde{I}_{n} A^{T} \widetilde{I}_{m}, \quad \text { for all } A \in \operatorname{Mat}_{m \times n}(k) .
$$

This variant of the transpose turns the first (second etc.) column of $A$ into the last (second last etc.) row of $A^{\widetilde{T}}$ but in reversed order.

\subsection{Companion matrices}

In this section we prove results which we need when dealing with the modules $C_{n}(\pi)$ and $C_{n}(\infty)$. So let $\pi$ be an irreducible polynomial over a perfect field $k$ of characteristic 2 and set $m:=\operatorname{deg}(\pi)$. Then $\pi$ has distinct roots $\epsilon_{1}, \ldots, \epsilon_{m}$ in a splitting field $\hat{k}$ over $k$. Set $K=k\left(\epsilon_{1}\right)$. 
Fix $n \geq 1$ and let $\mathcal{P} \in \operatorname{Mat}_{m n}(k)$ be the companion matrix of $\pi^{n}$. So $\mathcal{P}=$ $T_{0}+\sum_{i=1}^{m n} \lambda_{i-1} E_{i, n}$, where $\pi^{n}=\sum_{i=0}^{m n} \lambda_{i} x^{i}$. Now $\mathcal{P}$ is a non-derogatory matrix, meaning that its characteristic and minimal polynomials are equal (to $\pi^{n}$ ). In particular $\mathrm{C}_{\text {Mat }_{m n}(k)}(\mathcal{P})=k[\mathcal{P}]$ is isomorphic to $\frac{k[x]}{\left(\pi^{n}\right)}$. Next $\frac{k[x]}{\left(\pi^{n}\right)}$ is a commutative ring with a unique maximal ideal $(\pi)$. Also $\frac{k[x]}{(\pi)} \cong K$, and so $\frac{k[x]}{\left(\pi^{n}\right)} \cong \frac{K[t]}{\left(t^{n}\right)}$. Corresponding to this isomorphism, there is a unique expression (Jordan decomposition)

$$
\mathcal{P}=\mathcal{E}+\mathcal{N}, \quad \text { where } \mathcal{E} \text { has minimal polynomial } \pi \text { and } \mathcal{N} \text { is nilpotent. }
$$

The following lemma gives a more explicit version of this decomposition:

Lemma 3.3. Let $f(x) \in \hat{k}[x]$ be the unique polynomial of degree $<$ mn such that

$$
f(x) \equiv \epsilon_{i} \quad \bmod \left(x-\epsilon_{i}\right)^{n}, \quad i=1, \ldots, m .
$$

Then $f(x)$ has coefficients in $k$. Set $\mathcal{E}:=f(\mathcal{P})$ and $\mathcal{N}:=\mathcal{P}-\mathcal{E}$. Then $\mathcal{E} \mapsto \epsilon$ and $\mathcal{N} \mapsto t$ extends to a k-algebra isomorphism $\mathrm{C}_{\operatorname{Mat}_{m n}(k)}(\mathcal{P}) \cong \frac{K[t]}{\left(t^{n}\right)}$.

Set $\mathrm{C}(\mathcal{P})=\mathrm{C}_{\text {Mat }_{m n}(k)}(\mathcal{P})$ and identify $k(\mathcal{E})$ with $K$. Then each $A \in \mathrm{C}(\mathcal{P})$ can be written uniquely as $A=\sum_{i=0}^{n-1} \alpha_{i} \mathcal{N}^{i}$, where $\alpha_{i} \in K$ for $i=0, \ldots, n-1$. Set $|A|=$ $\min \left\{i \geq 0 \mid \alpha_{i} \neq 0\right\}$, if $A \neq 0$, and $|A|:=n$, otherwise. Then $|\cdot|$ is almost a Euclidean valuation, as

$$
\begin{aligned}
|A| & =0, \quad \text { if and only if } A \text { is a unit in } k[\mathcal{P}], \\
|A B| & =|A|+|B|, \quad \text { if } B \in \mathrm{C}(\mathcal{P}) \text { with } A B \neq 0, \text { and } \\
|A+B| & \geq \min \{|A|,|B|\}, \quad \text { with }>\text { if and only if }|A|=|B| \text { and } \alpha_{|A|}=\beta_{|B|} .
\end{aligned}
$$

Now $K$ is perfect as $k$ is perfect. So an easy consequence of Lemma 3.3 is that the set of squares in $\mathrm{C}(\mathcal{P})$ is $\left\{f\left(\mathcal{N}^{2}\right) \mid f \in K[x], \operatorname{deg}(f) \leq(n-1) / 2\right\}$. In particular the squares form a $k$-subspace of $\mathrm{C}(\mathcal{P})$ of dimension $m\left\lfloor\frac{n+1}{2}\right\rfloor$.

Lemma 3.4. Let $0 \leq v \leq w<n$ such that $w-v$ is even and let $A \in \mathrm{C}(\mathcal{P})$ with $|A|=v$. Then there are $U, V \in \mathrm{C}(\mathcal{P})$ such that $A U^{2}=\mathcal{N}^{w}+\mathcal{N}^{w+1} V^{2}$. This expression is unique, in the sense that $A W^{2}=\mathcal{N}^{w}+\mathcal{N}^{w+1} X^{2}$, with $W, X \in \mathrm{C}(\mathcal{P})$, if and only if $A W^{2}=A U^{2}$.

Proof. Write $A=\mathcal{N}^{v} P^{2}+\mathcal{N}^{v+1} Q^{2}$, with $P, Q \in K[\mathcal{N}]$. Then $P$ is a unit, as $|A|=v$. Set $U=P^{-1} \mathcal{N}^{(w-v) / 2}$. Then $A U^{2}=\mathcal{N}^{w}+\mathcal{N}^{w+1} V^{2}$, where $V=P^{-1} Q$.

Now suppose that $A W^{2}=\mathcal{N}^{w}+\mathcal{N}^{w+1} X^{2}$, for some $W, X \in \mathrm{C}(\mathcal{P})$. Then $A\left(U^{2}+W^{2}\right)=\mathcal{N}^{w+1}\left(V^{2}+X^{2}\right)$. It follows that $A\left(U^{2}+W^{2}\right)=0$, as otherwise we get the impossible integer equality $v+2|U+W|=w+1+2|V+X|$.

As a particular case, $A$ is a unit in $\mathrm{C}(\mathcal{P})$ if and only if there are $U, V \in \mathrm{C}(\mathcal{P})$ such that $A U^{2}=1+\mathcal{N} V^{2}$. In this case $U$ is a unit and $\mathcal{N} V^{2}$ is uniquely determined. 
Next, it is well-known that given an $n \times n$ matrix $M$ over any field $F$, there is a non-singular symmetric matrix $S$ in $\mathrm{T}_{\operatorname{Mat}_{n}(F)}(M)$. For example, if $J_{n}(\lambda)=\lambda I_{n}+T_{2}$ is an $n \times n$ Jordan block matrix, for $\lambda \in F$, then $\widetilde{I}_{n} J_{n}(\lambda)=J_{n}(\lambda)^{T} \widetilde{I}_{n}$. Moreover, it is a theorem of O. Taussky and H. Zassenhaus [5] that all matrices in $\mathrm{T}_{\operatorname{Mat}_{n}(F)}(M)$ are symmetric if and only if $M$ is non-derogatory. In particular all matrices in $\mathrm{T}_{\mathrm{Mat}_{m n}(k)}(\mathcal{P})$ are symmetric.

We can describe the symplectic forms on $C_{n}(\pi)$ and $C_{n}(\pi)^{2}$ in terms of an arbitrary non-singular matrix $\mathcal{T} \in \mathrm{T}_{\operatorname{Mat}_{m n}(k)}(\mathcal{P})$. However, in order to determine the existence of corresponding $G$-invariant quadratic forms (in Theorems 7.7 and 7.10) we must choose $\mathcal{T}$ carefully, as we now explain. This requires some Jordan form and Galois theory.

Let $\mathcal{J} \in \operatorname{Mat}_{m n}(\hat{k})$ be a Jordan normal form matrix of $\mathcal{P}$. So without loss of generality $\mathcal{J}=\operatorname{diag}\left(J_{n}\left(\epsilon_{1}\right), \ldots, J_{n}\left(\epsilon_{m}\right)\right)$, where $J_{n}(\epsilon):=\epsilon I_{n}+T_{2}$, for all $\epsilon \in \hat{k}$. Notice that $\operatorname{diag}\left(\widetilde{I}_{n}, \ldots, \widetilde{I}_{n}\right)$ is an invertible symmetric matrix in $\operatorname{T}_{\operatorname{Mat}_{m n}(k)}(\mathcal{J})$.

Lemma 3.5. There exists $V \in \mathrm{GL}_{m n}(\hat{k})$ such that $\mathcal{P}=V^{-1} \mathcal{J} V$ and $V^{T} \operatorname{diag}\left(\widetilde{I}_{n}, \ldots, \widetilde{I}_{n}\right) V$ has entries in $k$.

Proof. As $\mathcal{P}$ has Jordan form $\mathcal{J}$, there exists $V \in \mathrm{GL}_{m n}(\hat{k})$ such that $\mathcal{P}=V^{-1} \mathcal{J} V$. Let $V^{(j)} \in \operatorname{Mat}_{m n \times 1}(\hat{k})$ be the $j$-th column of $V$. As $\mathcal{P}=T_{-1}+\sum_{i=1}^{m n} \lambda_{i-1} E_{i, n}$, we have $V^{(j+1)}=V \cdot \mathcal{P}^{(j)}=\mathcal{J} \cdot V^{(j)}$, and thus $V^{(j)}=\mathcal{J}^{j-1} \cdot V^{(1)}$, for $j=2, \ldots m n$. In particular $V$ is uniquely determined by $V^{(1)}$.

Let $s \in\{1, \ldots, m\}$ and set $\mu_{s}:=V_{s n, 1}$. Note that for $j=1, \ldots m n$, the $(s n)$-th row of $\mathcal{J}^{j}$ has a single non-zero entry $\epsilon_{s}^{j}$ in the $(s n)$-th position. As $V^{(j)}=\mathcal{J}^{j-1} \cdot V^{(1)}$, the (sn)-th row of $V$ is $\mu_{s}\left(1, \epsilon_{s}, \ldots, \epsilon_{s}^{m n-1}\right)$. Since $V$ is non-singular, we deduce that $\mu_{s} \neq 0$.

Form the $n \times n$ upper-triangular Toeplitz matrix $X_{s}:=\sum_{i=1}^{n} V_{s n+1-i, 1} T_{i}$, for $s=$ $1, \ldots, m$. Then $X_{s}$ is non-singular, as it has diagonal entries $\mu_{s}$. Moreover it is clear that $\operatorname{diag}\left(X_{1}^{-1}, \ldots, X_{m}^{-1}\right) V^{(1)}$ is an $m n$-tuple with entry 1 at each position $n, 2 n, \ldots, m n$, and zeros elsewhere. Now $X_{s} \in \mathrm{C}_{\text {Mat }_{n}(\hat{k})}\left(J_{n}\left(\epsilon_{s}\right)\right)$. So replacing $V$ by $\operatorname{diag}\left(X_{1}^{-1}, \ldots, X_{m}^{-1}\right) V$, we may assume that $V^{(1)}=\sum_{s=1}^{m} E_{s n, 1}$ in $\operatorname{Mat}_{m n \times 1}(\hat{k})$.

Next there are $V_{(i, j)} \in \operatorname{Mat}_{n}(\hat{k})$ such that

$$
V=\left(\begin{array}{cccc}
V_{(1,1)} & V_{(1,2)} & \ldots & V_{(1, m)} \\
V_{(2,1)} & V_{(2,2)} & \ldots & V_{(2, m)} \\
\vdots & \vdots & & \vdots \\
V_{(m, 1)} & V_{(m, 2)} & \ldots & V_{(m, m)}
\end{array}\right)
$$

Also set $V_{(i)}=\left(\begin{array}{llll}V_{(i, 1)} & V_{(i, 2)} & \ldots & V_{(i, m)}\end{array}\right) \in \operatorname{Mat}_{n \times m n}(\hat{k})$, and let $V_{(i)}^{(j)}$ denote the $j$-th column of $V_{(i)}$. Then for $i=1, \ldots, m$ and $j=2, \ldots m n$ we have

$$
V_{(i)}^{(j)}=J_{n}(\epsilon)^{j-1} \cdot V_{(i)}^{(1)}=J_{n}(\epsilon)^{j-1} \cdot E_{n, 1} \cdot
$$


Now write $\operatorname{Gal}(\hat{k} / k)=\left\{\sigma_{1}, \ldots, \sigma_{m}\right\}$ where $\sigma_{i}\left(\epsilon_{1}\right)=\epsilon_{i}$, for $i=1, \ldots, m$. Acting on matrices entry-wise, $\sigma_{i}$ extends to a map $\operatorname{Mat}_{n \times m n}\left(k\left(\epsilon_{1}\right)\right) \rightarrow \operatorname{Mat}_{n \times m n}\left(k\left(\epsilon_{i}\right)\right)$. Then (6) implies that $V_{(i, j)}=\sigma_{i} V_{(1, j)}$, for $i, j=1, \ldots, m$.

Now write $V^{T} \operatorname{diag}\left(\widetilde{I}_{n}, \ldots, \widetilde{I}_{n}\right) V$ in $\operatorname{Mat}_{m}\left(\operatorname{Mat}_{n}(\hat{k})\right)$; the $(i, j)$-th block is

$$
\sum_{k=1}^{m} V_{(k, i)}^{T} \widetilde{I}_{n} V_{(k, j)}=\sum_{k=1}^{m}\left(\sigma_{k} V_{(1, i)}^{T}\right) \widetilde{I}_{n}\left(\sigma_{k} V_{(1, j)}\right)=\left(\sum_{k=1}^{n} \sigma_{k}\right) V_{(1, i)}^{T} \widetilde{I}_{n} V_{(1, j)}
$$

As this is $\operatorname{Gal}(\hat{k} / k)$-invariant, we conclude that $V^{T} \operatorname{diag}\left(\widetilde{I}_{n}, \ldots, \widetilde{I}_{n}\right) V$ has entries in $k$.

For the rest of the paper we take $V$ to be as in the statement of Lemma 3.5 and define

$$
\mathcal{T}=V^{T} \operatorname{diag}\left(\widetilde{I}_{n}, \ldots, \widetilde{I}_{n}\right) V
$$

So $\mathcal{T}$ is an invertible symmetric matrix in $\mathrm{T}_{\operatorname{Mat}_{m n}(k)}(\mathcal{P})$.

Corollary 3.6. $\mathrm{T}_{\mathrm{Mat}_{m n}(k)}(\mathcal{P})=\mathcal{T} k[\mathcal{P}]=V^{T} \operatorname{diag}\left(\widetilde{I}_{n}, \ldots, \widetilde{I}_{n}\right) k[\mathcal{J}] V$

Let $q \in k[x]$ be as in the statement of Lemma 3.3. Then for $i=1, \ldots, m$, we have $q\left(J_{n}\left(\epsilon_{i}\right)\right)=\epsilon_{i} I_{n}$ and $T_{2}=J_{n}\left(\epsilon_{i}\right)-\epsilon_{i} I_{n}$. As $\mathcal{P}=V^{-1} \mathcal{J} V$, it follows that

$$
\mathcal{E}=V^{-1} \operatorname{diag}\left(\epsilon_{1} I_{n}, \epsilon_{2} I_{n} \ldots, \epsilon_{m} I_{n}\right) V, \quad \text { and } \quad \mathcal{N}=V^{-1} \operatorname{diag}\left(T_{2}, T_{2} \ldots, T_{2}\right) V
$$

Notice that as each matrix in $\mathrm{T}(\mathcal{P})$ is symmetric we have

$$
X^{T} \mathcal{T} X=(\mathcal{T} X)^{T} X=\mathcal{T} X^{2}, \quad \text { for all } X \in \mathrm{C}(\mathcal{P})
$$

So $\mathcal{T}$ is determined up to multiplication by the square of a unit in $\mathrm{C}(\mathcal{P})$. Our next lemma depends crucially on our choice of $\mathcal{T}$.

Corollary 3.7. The alternating matrices in $\mathrm{T}_{\operatorname{Mat}_{m n}(k)}(\mathcal{P})$ are

$$
\begin{aligned}
\left\{\mathcal{T} C^{2} \mid C \in k[\mathcal{P}]\right\} & \text { if } n \text { is even, } \\
\left\{\mathcal{T} \mathcal{N} C^{2} \mid C \in k[\mathcal{P}]\right\} & \text { if } n \text { is odd. }
\end{aligned}
$$

Proof. By Lemma 3.2 and Corollary 3.6 the alternating matrices in $\mathrm{T}(\mathcal{P})$ correspond to the alternating matrices in $\operatorname{diag}\left(\widetilde{I}_{n}, \ldots, \widetilde{I}_{n}\right) k[\mathcal{J}]=\operatorname{diag}\left(\widetilde{I}_{n} k\left[J_{n}\left(\epsilon_{1}\right)\right], \ldots, \widetilde{I}_{n} k\left[J_{n}\left(\epsilon_{m}\right)\right]\right)$. Next note that $k\left[J_{n}\left(\epsilon_{j}\right)\right]$ is the space $\mathcal{T}_{n}\left(k\left(\epsilon_{j}\right)\right)$ of $n \times n$ upper-triangular Toeplitz matrices with entries in $k\left(\epsilon_{j}\right)$. Let $B=\sum_{i=0}^{n-1} \mu_{i} T_{1+i}$, with $\mu_{0}, \ldots, \mu_{n-1} \in k\left(\epsilon_{j}\right)$. Then $\widetilde{I}_{n} B=$ $\sum_{i=0}^{n-1} \mu_{i} H_{n+1+i}$.

Suppose first that $n$ is even. Then the diagonal of $\widetilde{I}_{n} B$ is $\left(0, \ldots, 0, \mu_{1}, \mu_{3}, \ldots, \mu_{n-1}\right)$. So $\widetilde{I}_{n} B$ is alternating if and only if $\mu_{1}=\mu_{3}=\cdots=\mu_{n-1}=0$. Also $T_{1+2 i}=\left(T_{2}^{i}\right)^{2}$, for $i \geq 0$ and $k\left(\epsilon_{j}\right)$ is a perfect field. So $\widetilde{I}_{n} B$ is alternating if and only if $B=\left(\sum_{i=0}^{n / 2} \sqrt{\mu_{2 i}} T_{2}^{i}\right)^{2}$ is 
a square in $k\left[J_{n}\left(\epsilon_{j}\right)\right]$. Then Corollary 3.6 implies that the alternating matrices in $\mathrm{T}(\mathcal{P})$ are $\left\{\mathcal{T} C^{2} \mid C \in k[\mathcal{P}]\right\}$.

Next suppose that $n$ is odd. Then the diagonal of $\widetilde{I}_{n} B$ is $\left(0, \ldots, 0, \mu_{0}, \mu_{2}, \ldots, \mu_{n-1}\right)$. So $\widetilde{I}_{n} B$ is alternating if and only if $\mu_{0}=\mu_{2}=\cdots=\mu_{n-1}=0$. It follows that $\widetilde{I}_{n} B$ is alternating if and only if $B=T_{2}\left(\sum_{i=0}^{(n-1) / 2} \sqrt{\mu_{1+2 i}} T_{2}^{i}\right)^{2}$. Then Corollary 3.6 implies that the alternating matrices in $\mathrm{T}(\mathcal{P})$ are $\left\{\mathcal{T} \mathcal{N} C^{2} \mid C \in k[\mathcal{P}]\right\}$.

\section{Indecomposable symplectic modules for the Klein-Four group}

The Klein-Four group is

$$
G=\left\{g_{1}, g_{2}, g_{3}, g_{4}\right\}
$$

where $g_{1}$ is the identity and $g_{2}, g_{3}, g_{4}=g_{2} g_{3}$ are involutions. Let $k$ be a perfect field of characteristic 2 . The indecomposable $k G$-modules are described in [1], where they are enumerated using the notation:

$$
D, A_{n}, B_{n}, C_{n}(\pi) \text { and } C_{n}(\infty) \text {. }
$$

Here $n \geq 1$ and $\pi$ is an irreducible polynomial over $k$. As mentioned in the introduction, we use $k_{G}$ to denote the trivial module $A_{0}=B_{0}$ and $k G$ to denote the regular module $D$.

Recall that an indecomposable symplectic $k G$-module is even-dimensional and either indecomposable as a $k G$-module or isomorphic to $N^{*} \oplus N$, for some indecomposable $k G$-module $N$. The modules $A_{n}$ and $B_{n}$ are odd-dimensional and dual to each other, and $C_{n}(\pi)$ and $C_{n}(\infty)$ are self-dual. So the $k G$-modules that are candidates for indecomposable symplectic modules are:

$$
\left(k_{G}\right)^{2}, k G,(k G)^{2}, A_{n} \oplus B_{n}, C_{n}(\pi), C_{n}(\pi)^{2}, C_{n}(\infty), C_{n}(\infty)^{2} .
$$

We discussed $\left(k_{G}\right)^{2}$ in the introduction. We deal with the remaining families in turn in the rest of the paper. For a module $M$ in each family we compute the endomorphism ring $\operatorname{End}_{k}(M)$ and describe all $G$-invariant symplectic forms on $M$. We then give a set of representatives $\operatorname{Isom}(M)$ for the isometry classes of symplectic forms. Finally, we determine which symplectic forms have an associated $G$-invariant quadratic form, and for each such symplectic form $B$ we give a set of representatives $\operatorname{QIsom}(M, B)$ for the isometry classes of $G$-invariant quadratic forms on $M$ which polarize to $B$.

\section{The regular module}

Temporarily let $G$ be an arbitrary finite group and see [3] for further details. We express $x \in k G$ as $x=\sum_{g \in G} x(g) g$, where $x(g) \in k$, for all $g \in G$. If $X \subseteq G$, then by $X^{+}$we mean $\sum_{x \in X} x \in k G$. The augmentation map is a $k$-algebra homomorphism 
||$: k G \rightarrow k,|x|=\sum_{g \in G} x(g)$, for all $x \in k G$. Its kernel is called the augmentation ideal of $k G$.

Let $B$ be the non-degenerate $G$-invariant symmetric bilinear form on $k G$ such that

$$
B(g, h)=\left\{\begin{array}{ll}
1_{k}, & \text { if } g=h \\
0_{k}, & \text { if } g \neq h
\end{array} \quad \text { for all } g, h \in G\right.
$$

It is well-known that $\operatorname{End}_{k G}(k G)$ can be identified with $k G^{o p}$. Here $x \in k G^{o p}$ acts by right-multiplication on $k G: y \mapsto y x$, for all $y \in k G$. The adjoint of $B$ is the contragredient map ${ }^{o}$ on $k G$ given by $g^{o}=g^{-1}$, for all $g \in G$. For each $x \in k G$, set $B_{x}(y, z):=B(y, z x)$, for all $y, z \in k G$. Then $\left\{B_{x} \mid x \in k G\right\}$ is the set of all $G$-invariant bilinear forms on $k G$. Note that $B_{x}(g, h)=x\left(h^{-1} g\right)$, for all $g, h \in G$. So the $G$-invariant symplectic forms are

$$
\left\{B_{x} \mid x \text { a unit in } k G, x\left(g_{1}\right)=0_{k} \text { and } x(g)=x\left(g^{-1}\right) \text {, for all } g \in G\right\} \text {, }
$$

with $g_{1}$ the identity in $G$. In the notation of Section 2.1 we have the $G$-invariant quadratic form $\Delta B(x)=|x|^{2}$, for all $x \in k G$. Note that $\Delta B$ polarizes to 0 . More generally fix a total order $g_{1}<g_{2}<\cdots<g_{n}$ on $G$. Let $B_{x}$ be a symplectic form on $k G$, for $x \in k G$, and let $\chi \in k$. Define $q_{\chi, x}:=\chi \Delta B+\Delta \nabla B_{x}$. Then

$$
q_{\chi, x}(y)=\chi|y|^{2}+\nabla B_{x}(y, y), \quad \text { for all } y \in k G
$$

So $q_{\chi, x}$ is a $G$-invariant quadratic form which polarizes to $B_{x}$. Conversely let $q$ be a $G$-invariant quadratic form on $k G$ which polarizes to $B_{x}$. Write $q\left(g_{1}\right)=\mu$, for some $\mu \in k$. Then by $G$-invariance $q(g)=\mu$, for all $g \in G$. It then follows from (1) that $q=q_{\mu, x}$.

We now return to our assumption that $G$ is the Klein-Four group. So $k G$ is a commutative ring, ${ }^{o}$ is the identity map, and $x^{2}=|x|^{2} g_{1}$, for all $x \in k G$. In particular $x$ is a unit in $k G$ if and only if $|x| \neq 0_{k}$.

Theorem 5.1. $\operatorname{Isom}(k G)=\left\{B_{x}\left|x \in k G, x\left(g_{1}\right)=0_{k},\right| x \mid=1_{k}\right\}$.

So if $k=\mathbb{F}_{q}$ then $|\operatorname{Isom}(k G)|=q^{2}$.

The G-invariant quadratic forms which polarize to $B_{x}$ are $\left\{q_{\chi, x} \mid \chi \in k\right\}$. Each $q_{\chi, x}$ is isometric to $q_{0, x}=\Delta \nabla B_{x}$.

Proof. By the discussion above, $\left\{B_{x}|x \in k G| x \mid, \neq 0_{k}, x\left(g_{1}\right)=0_{k}\right\}$ are the $G$-invariant symplectic forms on $k G$. Fix a symplectic form $B_{x}$ and let $u$ be a unit in $k G$. Then $u x u^{o}=u^{2} x=|u|^{2} x$. Now as $k$ is perfect we can choose $u$ such that $|u|^{2}=|x|^{-1}$. Then $\left|u^{2} x\right|=1_{k}$. So $B_{x}$ is isometric to $B_{y}$, for a unique $y \in k G$ such that $y\left(g_{1}\right)=0_{k}$ and $|y|=1_{k}$. This completes our description of $\operatorname{Isom}(k G)$.

Next let $q_{\chi, x}$ be a $G$-invariant quadratic form on $k G$, for $\chi \in k$. Let $u \in k G$ with $|u| \neq 0_{k}$. Then $q_{\chi, x}(u-)$ is a $G$-invariant quadratic form which polarizes to $B_{|u|^{2} x}$. So 
$\left\{q_{\chi, x}(u-)|u \in k G| u \mid,=1\right\}$ give all non-degenerate $G$-invariant quadratic forms on $k G$ which polarize to $B_{x}$ and which are isometric to $q_{\chi, x}$. Now if $|u|=1_{k}$, we get

$$
q_{\chi, x}(u-)=q_{\mu, x}, \quad \text { where } \mu=q_{\chi, x}(u) .
$$

Choose $u=\left(1_{k}+r\right) g_{1}+r\left(g_{2}+g_{3}+g_{4}\right)$, with $r=|x|^{-1} \chi$. Then $|u|=1_{k}$ and so by (1),

$$
\mu=\chi|u|^{2}+\left(x\left(g_{2}\right)+x\left(g_{3}\right)+x\left(g_{4}\right)\right)\left(\left(1_{k}+r\right) r+r^{2}\right)=0 .
$$

Thus we conclude that $q_{\chi, x}$ is isometric to $q_{0, x}$.

\subsection{Two copies of the regular module}

We next consider $(k G)^{2}$, which we identify with $\left\{\left(\begin{array}{l}x \\ y\end{array}\right) \mid x, y \in k G\right\}$. Recall the form $B$ on $k G$, given by (8), with trivial adjoint. The $G$-invariant bilinear forms on $(k G)^{2}$ can be written as $\left(\begin{array}{ll}a & b \\ c & d\end{array}\right)$, with $a, b, c, d \in k G$, where

$$
\left(\begin{array}{ll}
a & b \\
c & d
\end{array}\right)\left(\left(\begin{array}{l}
x_{1} \\
y_{1}
\end{array}\right),\left(\begin{array}{l}
x_{2} \\
y_{2}
\end{array}\right)\right):=B_{a}\left(x_{1}, x_{2}\right)+B_{b}\left(x_{1}, y_{2}\right)+B_{c}\left(y_{1}, x_{2}\right)+B_{d}\left(y_{1}, y_{2}\right)
$$

for all $\left(\begin{array}{c}x_{1} \\ y_{1}\end{array}\right),\left(\begin{array}{c}x_{2} \\ y_{2}\end{array}\right) \in(k G)^{2}$. Now $\left\{B_{x} \mid x \in \operatorname{sp}\left(g_{2}+g_{4}, g_{3}+g_{4}\right)\right\}$ is the space of degenerate $G$-invariant alternating forms on $k G$. Moreover the Jacobson radical of $\operatorname{End}_{G}(k G)=k G$ is the augmentation ideal of $k G$. So $\overline{\operatorname{End}_{G}(k G)} \cong k$. Now by Lemma 2.2, the indecomposable symplectic forms on $(k G)^{2}$ are $\left\{\left(\begin{array}{ll}a & b \\ b & d\end{array}\right)|a, b, d \in k G| b \mid, \neq 0_{k}, a, d \in \operatorname{sp}\left(g_{2}+g_{4}\right.\right.$, $\left.\left.g_{3}+g_{4}\right)\right\}$.

We denote the 'projective line' in the plane $\operatorname{sp}\left(g_{2}+g_{4}, g_{3}+g_{4}\right)$ by

$$
\mathbf{P}^{1}:=\left\{g_{3}+g_{4}\right\} \cup\left\{\left(g_{2}+g_{4}\right)+\lambda\left(g_{3}+g_{4}\right) \mid \lambda \in k\right\}
$$

Theorem 5.2. Isom $\left((k G)^{2}\right)=\left\{\left(\begin{array}{cc}0 & g_{1} \\ g_{1} & 0\end{array}\right),\left(\begin{array}{cc}x & g_{1} \\ g_{1} & 0\end{array}\right),\left(\begin{array}{cc}g_{2}+g_{4} & g_{1} \\ g_{1} & \mu\left(g_{3}+g_{4}\right)\end{array}\right) \mid \begin{array}{l}x \in \mathbf{P}^{1} \\ \mu \in k^{\times}\end{array}\right\}$. So $\left|\operatorname{Isom}\left((k G)^{2}\right)\right|=2 q+1$, if $k=\mathbb{F}_{q}$.

Proof. We fix an indecomposable $G$-invariant symplectic form $\left(\begin{array}{ll}a & b \\ b & d\end{array}\right)$ on $(k G)^{2}$. So $b$ is a unit in $k G$ and $a, d \in \operatorname{sp}\left(g_{2}+g_{4}, g_{3}+g_{4}\right)$. Let $\left(\begin{array}{cc}r & s \\ t & u\end{array}\right)$ be a unit in $\operatorname{End}_{G}\left((k G)^{2}\right)$, with non-zero 'determinant' $\chi:=r u+s t$. Then

$$
\left(\begin{array}{ll}
r & s \\
t & u
\end{array}\right)^{T}\left(\begin{array}{ll}
a & b \\
b & d
\end{array}\right)\left(\begin{array}{cc}
r & s \\
t & u
\end{array}\right)=\left(\begin{array}{cc}
r^{2} a+t^{2} d & \chi b+r s a+t u d \\
\chi b+r s a+t u d & s^{2} a+u^{2} d
\end{array}\right)
$$

So the isometry class of $\left(\begin{array}{ll}a & b \\ b & d\end{array}\right)$ is

$$
\left\{\left(\begin{array}{cc}
|r|^{2} a+|t|^{2} d & \chi b+r s a+t u d \\
\chi b+r s a+t u d & |s|^{2} a+|u|^{2} d
\end{array}\right) \mid r, s, t, u \in k G, r u+s t \neq 0\right\} .
$$




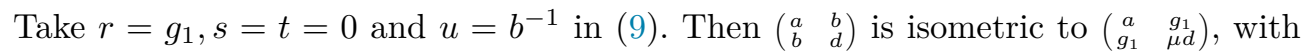
$\mu=|b|^{-2}$. There are three cases which we analyze in turn.

Case (i): Suppose that $a=d=0$. Then $\left((k G)^{2},\left(\begin{array}{ll}0 & b \\ b & 0\end{array}\right)\right)$ is isometric to the paired module. In particular all these forms are isometric to $\left(\begin{array}{cc}0 & g_{1} \\ g_{1} & 0\end{array}\right)$.

Case (ii): Next suppose that $a$ and $d$ are linearly dependent but not both 0 . Taking $r=u=0$ and $s=t=g_{1}$ in (9) we see that $\left(\begin{array}{ll}a & b \\ b & d\end{array}\right)$ is isometric to $\left(\begin{array}{ll}d & b \\ b & a\end{array}\right)$. So we can and do assume that $a \neq 0$ and $d=\epsilon^{2} a$, for some $\epsilon \in k$.

Now there is $\delta \in k$ such that $\delta^{2} a=g_{3}+g_{4}$ or $\delta^{2} a=\left(g_{2}+g_{4}\right)+\lambda\left(g_{3}+g_{4}\right)$, for some $\lambda \in k$. Choose $r=\delta g_{1}, s=\epsilon g_{1}, t=0, u=g_{1}$ in (9), we see that $\left(\begin{array}{ll}a & b \\ b & d\end{array}\right)$ is isometric to $\left(\begin{array}{cc}g_{3}+g_{4} & \delta b+\delta \epsilon a \\ \delta b+\delta \epsilon a & 0\end{array}\right)$ or $\left(\begin{array}{cc}\left(g_{2}+g_{4}\right)+\lambda\left(g_{3}+g_{4}\right) & \delta b+\delta \epsilon a \\ \delta b & +\delta \epsilon a\end{array}\right)$. These forms are in turn isometric to

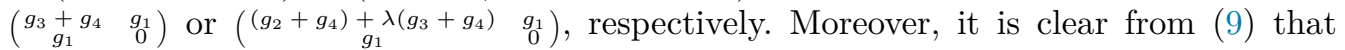
these forms are pairwise non-isometric.

Case (iii): Finally suppose that $a$ and $d$ are linearly independent. Then there are $\alpha, \beta, \gamma, \delta \in k$, with $\alpha \gamma+\beta^{2} \neq 0_{k}$ such that

$$
\alpha^{2} a+\gamma^{2} d=g_{2}+g_{4} \quad \text { and } \quad \beta^{2} a+\delta^{2} d=g_{3}+g_{4}
$$

Taking $r=\alpha g_{1}, s=\beta g_{1}, t=\gamma g_{1}, u=\delta g_{1}$ in (9), and with $\chi=\alpha \gamma+\beta^{2}$, we see that $\left(\begin{array}{ll}a & b \\ b & d\end{array}\right)$ is isometric to $\left(\begin{array}{cc}g_{2}+g_{4} & \chi b+\alpha \beta a+\gamma \delta d \\ \chi b+\alpha \beta a+\gamma \delta d & g_{3}+g_{4}\end{array}\right)$. This is in turn isometric to $\left(\begin{array}{cc}g_{2}+g_{4} & g_{1} \\ g_{1} & \mu\left(g_{3}+g_{4}\right)\end{array}\right)$ with $\mu^{2}=|\chi b+\alpha \beta a+\gamma \delta d|=\chi|b|$. In particular $\mu \neq 0_{k}$.

Now suppose that $\left(\begin{array}{cc}g_{g_{1}}+g_{4} & \mu\left(g_{3}+g_{4}\right)\end{array}\right)$ is isometric to $\left(\begin{array}{l}g_{2}+g_{4} \\ g_{1} \\ \mu^{\prime}\left(g_{3}+g_{4}\right)\end{array}\right)$ with $\mu^{\prime} \in k^{\times}$, via an isometry $\left(\begin{array}{cc}r & s \\ t & u\end{array}\right)$. As by assumption $|r|^{2}|u|^{2} \neq|s|^{2}|t|^{2}$ in $k$, the equalities

$$
\begin{aligned}
& |r|^{2}\left(g_{2}+g_{4}\right)+|t|^{2} \mu\left(g_{3}+g_{4}\right)=\left(g_{2}+g_{4}\right) \\
& |s|^{2}\left(g_{2}+g_{4}\right)+|u|^{2} \mu\left(g_{3}+g_{4}\right)=\mu^{\prime}\left(g_{3}+g_{4}\right)
\end{aligned}
$$

imply that $|r|=1_{k},|u|^{2} \mu=\mu^{\prime}$ and $|s|=|t|=0_{k}$. But also

$$
(r u+s t) g_{1}+t u\left(g_{2}+g_{4}\right)+r s \mu\left(g_{3}+g_{4}\right)=g_{1}
$$

So $|r||u|=1_{k}$. As $|r|=1_{k}$ we get $|u|=1_{k}$. We conclude that $\mu^{\prime}=\mu$.

In order to describe the $G$-invariant quadratic forms, fix a symplectic form $\left(\begin{array}{ll}a & b \\ b & d\end{array}\right)$. Then for all $\alpha, \delta \in k$, there is a $G$-invariant quadratic form which polarizes to $\left(\begin{array}{ll}a & b \\ b & d\end{array}\right)$ :

$$
q_{\left(\begin{array}{l}
\alpha \\
\delta
\end{array}\right),\left(\begin{array}{ll}
a & b \\
b & d
\end{array}\right)}\left(\begin{array}{l}
x \\
y
\end{array}\right):=q_{\alpha, a}(x)+B_{b}(x, y)+q_{\delta, d}(y), \quad \text { for all } x, y \in k G
$$

So $q_{\left(\begin{array}{ll}\alpha \\ \delta\end{array}\right),\left(\begin{array}{ll}a & b \\ b & d\end{array}\right)}=\Delta\left(\begin{array}{cc}\alpha g_{1} & 0 \\ 0 & \delta g_{1}\end{array}\right)+\Delta \nabla\left(\begin{array}{ll}a & b \\ b & d\end{array}\right)$, in the notation of Section 2.1 .

Theorem 5.3. $\left\{q_{\left(\begin{array}{ll}\alpha \\ \delta\end{array}\right),\left(\begin{array}{ll}a & b \\ b & d\end{array}\right)} \mid \alpha, \delta \in k\right\}$ are the G-invariant quadratic forms on $(k G)^{2}$ which polarize to $\left(\begin{array}{ll}a & b \\ b & d\end{array}\right)$. Each of these is isometric to $\left.q_{\left(\begin{array}{l}0 \\ 0\end{array}\right),\left(\begin{array}{ll}a & b \\ b & d\end{array}\right)}\right)=\Delta \nabla\left(\begin{array}{ll}a & b \\ b & d\end{array}\right)$. 
Proof. Let $q$ be a $G$-invariant quadratic form on $k G$ which polarizes to $\left(\begin{array}{ll}a & b \\ b & d\end{array}\right)$, and set $\alpha:=q\left(\begin{array}{c}g_{1} \\ 0\end{array}\right)$ and $\delta:=q\left(\begin{array}{l}0 \\ g_{1}\end{array}\right)$. The $G$-invariance of $q$ implies that $q\left(\begin{array}{l}g \\ 0\end{array}\right)=\alpha$ and $q\left(\begin{array}{l}0 \\ g\end{array}\right)=\delta$, for all $g \in G$. Now the diagonal of the upper-triangular form of $\left(\begin{array}{ll}a & b \\ b & d\end{array}\right)$ is the $G$-invariant quadratic form

$$
\Delta \nabla\left(\begin{array}{ll}
a & b \\
b & d
\end{array}\right)\left(\begin{array}{l}
x \\
y
\end{array}\right)=q_{0, a}(x)+B_{b}(x, y)+q_{0, d}(y), \quad \text { for all } x, y \in k G
$$

It then follows from (1) that $q=q_{\left(\begin{array}{l}\alpha \\ \delta\end{array}\right),\left(\begin{array}{ll}a & b \\ b & d\end{array}\right)}$.

To describe the isometry classes of quadratic forms, let $\left(\begin{array}{cc}r & s \\ t & u\end{array}\right)$ be a unit in $\operatorname{End}_{G}\left((k G)^{2}\right)$. Then $q_{\left(\begin{array}{ll}\alpha \\ \delta\end{array}\right),\left(\begin{array}{ll}a & b \\ b & d\end{array}\right)}\left(\left(\begin{array}{ll}r & s \\ t & u\end{array}\right)-\right)$ is a $G$-invariant quadratic form on $(k G)^{2}$ which polarizes to $\left(\begin{array}{cc}|r|^{2} a+|t|^{2} d & \chi b+r s a+t u d \\ \chi b+r s a+t u d & |s|^{2} a+|u|^{2} d\end{array}\right)$, according to (9). Now consider the unit with $r=u=g_{1}$ and $s=\mu G^{+}, t=\nu G^{+}$, for $\mu, \nu \in k$. This fixes $\left(\begin{array}{ll}a & b \\ b & d\end{array}\right)$, as $\chi=1_{k}$, $s a=\mu|a| G^{+}=0$ and $t d=\mu|d| G^{+}=0$. Now

$$
\Delta \nabla\left(\begin{array}{ll}
a & b \\
b & d
\end{array}\right)\left(\left(\begin{array}{cc}
g_{1} & \mu G^{+} \\
\nu G^{+} & g_{1}
\end{array}\right)\left(\begin{array}{c}
g_{1} \\
0
\end{array}\right)\right)=q_{0, a}\left(g_{1}\right)+B_{b}\left(g_{1}, \nu G^{+}\right)+q_{0, d}\left(\nu G^{+}\right)=\nu|b|
$$

Likewise $\Delta \nabla\left(\begin{array}{ll}a & b \\ b & d\end{array}\right)\left(\left(\begin{array}{cc}g_{1} & \mu G^{+} \\ \nu G^{+} & g_{1}\end{array}\right)\left(\begin{array}{c}0 \\ g_{1}\end{array}\right)\right)=\mu|b|$. Taking $\nu=\alpha|b|^{-1}$ and $\mu=\delta|b|^{-1}$, we see that $q_{\left(\begin{array}{l}\alpha \\ \delta\end{array}\right),\left(\begin{array}{ll}a & b \\ b & d\end{array}\right)}$ is isometric to $\Delta \nabla\left(\begin{array}{ll}a & b \\ b & d\end{array}\right)$.

\section{The modules $\boldsymbol{A}_{n} \oplus \boldsymbol{B}_{n}$}

We follow the description of $A_{n}$ and $B_{n}$ given in [1]. First note that $\operatorname{dim}_{k}\left(A_{n}\right)=$ $\operatorname{dim}_{k}\left(B_{n}\right)=2 n+1$ is odd. So neither $A_{n}$ nor $B_{n}$ afford a $G$-invariant symplectic form. Set $n_{2}:=g_{1}+g_{2}$ and $n_{3}:=g_{1}+g_{3}$ in $k G$. Then $n_{2}$ and $n_{3}$ act on a basis $\left(a_{1}, \ldots, a_{n}, b_{0}, \ldots, b_{n}\right)$ of $A_{n}$ as follows:

$$
\begin{array}{rlrl}
n_{2} a_{i} & =n_{3} a_{i}=0, & & \text { for all } i=1, \ldots, n . \\
n_{2} b_{0}=n_{3} b_{n}=0, & n_{2} b_{j}=n_{3} b_{j-1}=a_{j}, & \text { for all } j=1, \ldots, n .
\end{array}
$$

So with respect to the basis

$$
n_{2}=\left(\begin{array}{cc}
0 & T_{2} \\
0 & 0
\end{array}\right) \text { and } n_{3}=\left(\begin{array}{cc}
0 & T_{1} \\
0 & 0
\end{array}\right) .
$$

Here $T_{2}$ and $T_{1}$ are $n \times(n+1)$ Toeplitz matrices with 1 's on the superdiagonal and diagonal, respectively. Likewise $n_{2}$ and $n_{3}$ act on a basis $\left(a_{0}, \ldots, a_{n}, b_{1}, \ldots, b_{n}\right)$ of $B_{n}$ as follows:

$$
\begin{aligned}
& n_{2} a_{i}=n_{3} a_{i}=0, \quad \text { for all } i=0, \ldots, n \text {. } \\
& n_{2} b_{1}=a_{0}, \quad n_{3} b_{n}=a_{n}, \quad n_{2} b_{j+1}=n_{3} b_{j}=a_{j}, \quad \text { for all } j=1, \ldots, n-1 \text {. }
\end{aligned}
$$

So with respect to the basis 


$$
n_{2}=\left(\begin{array}{cc}
0 & T_{1} \\
0 & 0
\end{array}\right) \text { and } n_{3}=\left(\begin{array}{cc}
0 & T_{0} \\
0 & 0
\end{array}\right)
$$

where $T_{1}$ and $T_{0}$ are $(n+1) \times n$ Toeplitz matrices with 1 's on the diagonal and subdiagonal, respectively.

Lemma 6.1. $A_{n}^{*} \cong B_{n}$, for all $n \geq 0$.

Proof. By inspection if $n_{2}$ maps $b_{i}$ to $a_{j}$ in $A_{n}$, then $n_{2}$ maps $b_{n-j+1}$ to $a_{n-i}$ in $B_{n}$. The same is true of $n_{3}$. It follows that the map $b_{n-i+1} \mapsto a_{i}^{T}$, for $i=1, \ldots, n$, and $a_{n-j} \mapsto b_{j}^{T}$, for $j=0, \ldots, n$, extends to a $k G$-isomorphism $B_{n} \cong A_{n}^{*}$. Equivalently

$$
\widetilde{I}_{2 n+1}\left(\begin{array}{cc}
0 & T_{2} \\
0 & 0
\end{array}\right)^{T} \widetilde{I}_{2 n+1}=\left(\begin{array}{cc}
0 & T_{1} \\
0 & 0
\end{array}\right) \quad \text { and } \quad \widetilde{I}_{2 n+1}\left(\begin{array}{cc}
0 & T_{1} \\
0 & 0
\end{array}\right)^{T} \widetilde{I}_{2 n+1}=\left(\begin{array}{cc}
0 & T_{0} \\
0 & 0
\end{array}\right)
$$

We represent the elements of $A_{n} \oplus B_{n}$ by column vectors $\left(\begin{array}{l}a \\ b\end{array}\right)$, with $a \in A_{n}$ and $b \in B_{n}$, and we use the following ordered basis of $A_{n} \oplus B_{n}$ :

$$
\mathcal{B}:=\left\{\left(\begin{array}{c}
a_{1} \\
0
\end{array}\right), \ldots,\left(\begin{array}{c}
a_{n} \\
0
\end{array}\right),\left(\begin{array}{c}
0 \\
a_{0}
\end{array}\right), \ldots,\left(\begin{array}{c}
0 \\
a_{n}
\end{array}\right),\left(\begin{array}{c}
b_{0} \\
0
\end{array}\right), \ldots,\left(\begin{array}{c}
b_{n} \\
0
\end{array}\right),\left(\begin{array}{c}
0 \\
b_{1}
\end{array}\right), \ldots,\left(\begin{array}{c}
0 \\
b_{n}
\end{array}\right)\right\} .
$$

So the action of $k G$ on $A_{n} \oplus B_{n}$ is defined by the following matrices, with respect to $\mathcal{B}$ :

$$
n_{2}=\left(\begin{array}{cc}
0 & T_{2} \\
0 & 0
\end{array}\right) \quad \text { and } \quad n_{3}=\left(\begin{array}{cc}
0 & I_{2 n+1}+E_{n+1} \\
0 & 0
\end{array}\right)
$$

Here $T_{2}$ is the $(2 n+1) \times(2 n+1)$ Toeplitz matrix with 1 's on the superdiagonal and $I_{2 n+1}+E_{n+1}=\operatorname{diag}\left(1^{n}, 0,1^{n}\right)$. In fact in the following we describe all endomorphisms and forms on $A_{n} \oplus B_{n}$ with respect to the basis $\mathcal{B}$.

Now recall that $\mathcal{T}_{m \times n}(k)$ is the space of $m \times n$ Toeplitz matrices and that $M^{\widetilde{T}}=\widetilde{I} M^{T} \widetilde{I}$ is the exchange-transpose operation, as defined in (4).

Lemma 6.2. $\operatorname{End}_{G}\left(A_{n} \oplus B_{n}\right)=\left\{\left(\begin{array}{cccc}\alpha I_{n} & X & \star & \star \\ \cdot & \beta I_{n+1} & \star & \star \\ \cdot & \vdots & \alpha I_{n+1} & X^{T} \\ \cdot & \cdot & \cdot & \beta I_{n}\end{array}\right) \mid \begin{array}{c}\alpha, \beta \in k, \\ X \in \mathcal{T}_{n \times(n+1)}\end{array}\right\}$.

Proof. Let $A, B, C, D \in \operatorname{Mat}_{2 n+1}(k)$. Then $\left(\begin{array}{cc}A & B \\ C & D\end{array}\right) \in \operatorname{End}_{G}\left(A_{n} \oplus B_{n}\right)$ if and only if

$$
\begin{aligned}
\text { (i) } & A \cdot T_{2}=T_{2} \cdot D \\
\text { (ii) } & C \cdot T_{2}=0=T_{2} \cdot C \\
\text { (iii) } & A+A \cdot E_{n+1}=D+E_{n+1} \cdot D \\
\text { (iv) } & C+C \cdot E_{n+1}=0=C+E_{n+1} \cdot C
\end{aligned}
$$

First note that (ii) and (iv) hold if and only if $C=0$. Next (i) holds precisely if for all $k \neq 2 n+1$ and $l \neq 1$ we have 


$$
A_{k, l-1}=D_{k+1, l} \text { and } A_{2 n+1, k}=0=D_{l, 1} .
$$

Finally (iii) holds if and only if for all $k, l \neq n+1$ we have

$$
A_{k, l}=D_{k, l} \text { and } A_{n+1, l}=0=D_{k, n+1} .
$$

Hence the matrices described in the statement indeed belong to $\operatorname{End}_{G}\left(A_{n} \oplus B_{n}\right)$.

Now suppose that $C=0$ and (11) and (12) are satisfied. Then $A=\left(\begin{array}{ccc}X_{1} & a_{1} & X_{2} \\ \dot{C}_{3} & a_{2} & \dot{X} \\ X_{3} & a_{3} & X_{4}\end{array}\right)$ and $D=\left(\begin{array}{ccc}X_{1} & \cdot & X_{2} \\ d_{1} & d_{2} & d_{3} \\ X_{3} & \cdot & X_{4}\end{array}\right)$, by (12). Here each $X_{i}$ is an $n \times n$ matrix, $a_{2}, d_{2} \in k$, and $a_{1}, a_{3}$ and $d_{1}, d_{3}$ are columns and rows of length $n$, respectively. Let $k, l \in\{1, \ldots, 2 n+1\}$. Then $A_{k, l}=D_{k+1, l+1}$, unless $k=2 n+1$ or $l=2 n+1$, by (11). Also $A_{k, l}=D_{k, l}$, unless $k=n+1$ or $l=n+1$, by (12). In particular $A_{k, l}=A_{k+1, l+1}$, unless $k, l \in\{n, 2 n+1\}$ and $D_{k, l}=D_{k+1, l+1}$, unless $k, l \in\{n+1,2 n+1\}$. Therefore the following matrices are Toeplitz-matrices

$$
\left(\begin{array}{ll}
X_{1} & \dot{d_{2}} \\
d_{1} & \dot{d}_{2}
\end{array}\right),\left(\begin{array}{cc}
a_{1} & X_{2} \\
\star & d_{3}
\end{array}\right),\left(\begin{array}{l}
\dot{X_{3}}
\end{array}\right),\left(\begin{array}{ll}
a_{2} & \dot{X}_{4} \\
a_{3} & X_{4}
\end{array}\right) .
$$

Above we have seen that the first column of $D$ is zero except possibly for the first entry, which we call $\alpha$. Also the last row of $A$ is zero except possibly for the last entry, which we call $\beta$. Thus it follows that $X_{1}=\alpha I, d_{1}=0, d_{2}=\alpha, X_{3}=0, a_{2}=\beta, a_{3}=0$, $X_{4}=\beta I$ and $d_{3}=a_{2}^{\widetilde{T}}$. Finally consider the $n \times(n+1)$ matrix $X:=\left(\begin{array}{ll}a_{1} & X_{2}\end{array}\right)$. Then $X$ is a Toeplitz matrix and $X^{\widetilde{T}}=\left(\begin{array}{l}X_{2} \\ d_{3}\end{array}\right)$. This completes the proof.

Next recall that $\mathcal{H}_{m \times n}(k)$ is the space of $m \times n$ Hankel matrices.

Lemma 6.3. The $G$-invariant alternating forms on $A_{n} \oplus B_{n}$ are

$$
\left\{\left(\begin{array}{cc}
0 & B \\
B^{T} & D
\end{array}\right) \mid B=\left(\begin{array}{cc}
0 & \alpha \widetilde{I}_{n} \\
\alpha \widetilde{I}_{n+1} & H
\end{array}\right), \begin{array}{c}
\alpha \in k^{\times}, H \in \mathcal{H}_{(n+1) \times n}(k) \\
D \in \operatorname{Mat}_{2 n+1}(k) \text { alternating }
\end{array}\right\}
$$

Proof. The proof of Lemma 6.1 can be interpreted as showing that $\widetilde{I}_{4 n+2}$ is the Gram matrix, with respect to $\mathcal{B}$, of a $G$-invariant symplectic form on $A_{n} \oplus B_{n}$ (this is equivalent to the identities $g_{2}^{T} \cdot \widetilde{I}_{4 n+2} \cdot g_{2}=\widetilde{I}_{4 n+2}$ and $g_{3}{ }^{T} \cdot \widetilde{I}_{4 n+2} \cdot g_{3}=\widetilde{I}_{4 n+2}$ ). It follows from this that $\left\{\widetilde{I}_{4 n+2} \cdot A \mid A \in \operatorname{End}_{G}\left(A_{n} \oplus B_{n}\right)\right\}$ is the set of all $G$-invariant bilinear forms on $A_{n} \oplus B_{n}$. Now in the statement of Lemma $6.2, X$ is an $n \times(n+1)$ Toeplitz matrix. Set $H=\widetilde{I}_{n} X$. Then $H^{T}=X \widetilde{I}_{n}=\widetilde{I}_{n+1} X^{\widetilde{T}}$. Moreover $H$ is an $(n+1) \times n$ Hankel matrix. The Lemma follows from these facts.

Recall that $\mathcal{M} \mathcal{H}_{m \times n}(k)$ is the set of monic $m \times n$ Hankel matrices. We can now prove the main theorem of this section.

Theorem 6.4. $\operatorname{Isom}\left(A_{n} \oplus B_{n}\right)=\left\{\left(\begin{array}{cc}0 & B \\ B^{T} & 0\end{array}\right) \mid B=\left(\begin{array}{cc}0 & \widetilde{I}_{n} \\ \widetilde{I}_{n+1} & H\end{array}\right), H \in \mathcal{M} \mathcal{H}_{(n+1) \times n}(k)\right\}$. 
Proof. By Lemma 6.3 each indecomposable symplectic form on $A_{n} \oplus B_{n}$ has a matrix $\left(\begin{array}{cc}0 & A \\ A^{T} & D\end{array}\right)$ where $A, D \in \operatorname{Mat}_{(2 n+1)}(k)$, with $D$ alternating and $A=\left(\begin{array}{cc}0 & \mu \widetilde{I}_{n} \\ \mu \widetilde{I}_{n+1} & H^{\prime}\end{array}\right)$ for some $\mu \in k^{\times}$and $H^{\prime} \in \mathcal{H}_{(n+1) \times n}(k)$. By Lemma 6.2 each unit in $\operatorname{End}_{G}\left(A_{n} \oplus B_{n}\right)$ has the form $\left(\begin{array}{cc}M_{1} & M_{2} \\ 0 & M_{4}\end{array}\right)$, where $M_{1}=\left(\begin{array}{cc}\alpha I_{n} & X \\ 0 & \beta I_{n+1}\end{array}\right), M_{4}=\left(\begin{array}{cc}\alpha I_{n+1} & X_{0}^{\widetilde{T}} \\ 0 & \beta I_{n}\end{array}\right)$, for $\alpha, \beta \in k^{\times}$and $X \in \mathcal{T}_{n \times n+1}(k)$, and $M_{2} \in \operatorname{Mat}_{2 n+1}(k)$. Then

$$
\left(\begin{array}{cc}
M_{1} & M_{2} \\
0 & M_{4}
\end{array}\right)^{T}\left(\begin{array}{cc}
0 & A \\
A^{T} & D
\end{array}\right)\left(\begin{array}{cc}
M_{1} & M_{2} \\
0 & M_{4}
\end{array}\right)=\left(\begin{array}{cc}
0 & M_{1}^{T} A M_{4} \\
M_{4}^{T} A^{T} M_{1} & M_{2}^{T} A M_{4}+M_{4}^{T} A^{T} M_{2}+M_{4}^{T} D M_{4}
\end{array}\right)
$$

As $D$ is symmetric and hollow there is some $Z \in \operatorname{Mat}_{2 n+1}(k)$ such that $D=Z+Z^{T}$. Applying (13) with $M_{1}=M_{4}=I_{2 n+1}$ and $M_{2}=A^{-T} Z$, we see that $\left(\begin{array}{cc}0 & A \\ A^{T} & D\end{array}\right)$ is isometric to $\left(\begin{array}{cc}0 & A \\ A^{T} & 0\end{array}\right)$. So from now on we assume that $D=0$.

Now the previous paragraph and (13) show that $\left(\begin{array}{cc}0 & A \\ A^{T} & 0\end{array}\right)$ is isometric to $\left(\begin{array}{cc}0 & B \\ B^{T} & 0\end{array}\right)$ if and only if $B=M_{1}^{T} A M_{4}$, where $M_{1}$ and $M_{4}$ are of the form given above. Now

$$
M_{1}^{T} A M_{4}=\left(\begin{array}{cc}
0 & \alpha \beta \mu \widetilde{I}_{n} \\
\alpha \beta \mu \widetilde{I}_{n+1} & \beta \mu\left(X^{T} \widetilde{I}_{n}+\widetilde{I}_{n+1} X^{\widetilde{T}}\right)+\beta^{2} H^{\prime}
\end{array}\right)=\left(\begin{array}{cc}
0 & \alpha \beta \mu \widetilde{I}_{n} \\
\alpha \beta \mu \widetilde{I}_{n+1} & \beta^{2} H^{\prime}
\end{array}\right)
$$

as $\widetilde{I}_{n+1} X^{\widetilde{T}}=X^{T} \widetilde{I}_{n}$. Next there is $\beta \in k^{\times}$such that $H:=\beta^{2} H^{\prime}$ lies in $\mathcal{M} \mathcal{H}_{(n+1) \times n}(k)$. Moreover note that $\beta$ is unique if $H^{\prime} \neq 0$. Now set $\alpha:=(\beta \mu)^{-1} \in k^{\times}$. Then $M_{1}^{T} A M_{4}=$ $\left(\begin{array}{cc}0 & \widetilde{I}_{n} \\ \tilde{I}_{n+1} & H\end{array}\right)$. Finally $H$ is clearly determined by the isometry class of $\left(\begin{array}{cc}0 & A \\ A^{T} & D\end{array}\right)$.

Corollary 6.5. If $k=\mathbb{F}_{q}$, then $\left|\operatorname{Isom}\left(A_{n} \oplus B_{n}\right)\right|=1+\frac{q^{2 n}-1}{q-1}$.

We use our next lemma to describe the isometry classes of $G$-invariant quadratic forms on $A_{n} \oplus B_{n}$, and reuse it for the modules $C_{n}(\pi)$ and $C_{n}(\pi)^{2}$. Recall that $E_{t}$ is a square matrix with $(i, j)$-th entry $\delta_{i, t} \delta_{j, t}$ and $\mathcal{P}(k)$ is a set of representatives for the distinct cosets of the additive subgroup $\left\{\lambda^{2}+\lambda \mid \lambda \in k\right\}$ in $(k,+)$. Also we write $A \asymp B$ for square matrices $A, B$ if $A+B$ is alternating.

Lemma 6.6. Let $M$ be a $k G$-module of dimension $2 n$ such that with respect to a given basis $\mathcal{B}$ we have $\left(\begin{array}{cc}I_{n} & X \\ 0 & I_{n}\end{array}\right) \in \operatorname{End}_{G}(M)$, for all $X \in \operatorname{Mat}_{n}(k)$. Also let $\Delta\left(\begin{array}{cc}A & B \\ 0 & D\end{array}\right)$ be a G-invariant quadratic form on $M$, where $B \in \mathrm{GL}_{n}(k)$ and $A, D \in \operatorname{Diag}_{n}(k)$.

(i) If $A=0$, then $\Delta\left(\begin{array}{ll}0 & B \\ 0 & D\end{array}\right)$ is isometric to $\Delta\left(\begin{array}{cc}0 & B \\ 0 & 0\end{array}\right)$.

(ii) If $A \neq 0$, there exists $t \in\{1, \ldots, n\}$ such that $\left(B^{-1} A B^{-T}\right)_{t, t} \neq 0$. Then $\Delta\left(\begin{array}{cc}A & B \\ 0 & D\end{array}\right)$ is isometric to $\Delta\left(\begin{array}{cc}A & B \\ 0 & \tau E_{t}\end{array}\right)$ for a unique $\tau \in\left(B^{-1} A B^{-T}\right)_{t, t}^{-1} \mathcal{P}(k)$. 
Proof. We note that $\Delta\left(\begin{array}{cc}A & B \\ 0 & D\end{array}\right)$ polarizes to the $G$-invariant symplectic form $\left(\begin{array}{cc}0 & B \\ B^{T} & 0\end{array}\right)$. Let $X \in \operatorname{Mat}_{n}(k)$. Then by assumption $\left(\begin{array}{cc}I_{n} & X \\ 0 & I_{n}\end{array}\right)$ is a unit in $\operatorname{End}_{G}(M)$ and

$$
\left(\begin{array}{cc}
I_{n} & X \\
0 & I_{n}
\end{array}\right)^{T}\left(\begin{array}{cc}
A & B \\
0 & D
\end{array}\right)\left(\begin{array}{cc}
I_{n} & X \\
0 & I_{n}
\end{array}\right)=\left(\begin{array}{cc}
A & A X+B \\
X^{T} A & X^{T} A X+X^{T} B+D
\end{array}\right)
$$

First suppose that $A=0$. Choose $X=B^{-T} D$. Then $X^{T} A X+X^{T} B+D=0$. So $\left(\begin{array}{ll}A & B \\ 0 & D\end{array}\right)$ and $\left(\begin{array}{ll}0 & B \\ 0 & 0\end{array}\right)$ are isometric. In particular part (i) holds.

Next suppose that $A \neq 0$ and set $\widetilde{A}:=B^{-1} A B^{-T}$. Then $\widetilde{A}$ is not hollow, by Lemma 3.2. Let $t \in\{1, \ldots, n\}$ be such that $\eta:=\widetilde{A}_{t, t}$ is not zero.

We construct a symmetric matrix $L \in \operatorname{Mat}_{n}(k)$ such that $L \widetilde{A} L+L+\tau E_{t} \asymp D$, for some $\tau \in \eta^{-1} \mathcal{P}(k)$. First set $L_{i, j}=0$, for all $i, j \in\{1, \ldots, n\} \backslash\{t\}$. Then by Lemma 3.2 we have

$$
\left(L \widetilde{A} L+L+\tau E_{t}\right)_{i, i}= \begin{cases}\eta L_{t, i}^{2}, & \text { if } i \neq t . \\ \sum_{j=1}^{n} \widetilde{A}_{j, j} L_{j, t}^{2}+L_{t, t}+\tau, & \text { if } i=t .\end{cases}
$$

As $k$ is perfect, we can choose $L_{t, i}=L_{i, t} \in k$ such that $\eta L_{t, i}^{2}=D_{i, i}$, for $i \neq t$. We complete the construction of $L$ by making the unique choice of $L_{t, t}, \tau \in k$ such that $\tau \eta \in \mathcal{P}(k)$ and

$$
\sum_{\substack{j=1 \\ j \neq t}}^{n} \widetilde{A}_{j, j} L_{j, t}^{2}+\eta^{-1}\left(\left(\eta L_{t, t}\right)^{2}+\eta L_{t, t}+\tau \eta\right)=D_{t, t}
$$

Now set $X=B^{-T} L$. Then $X^{T} A X+X^{T} B+D=L \widetilde{A} L+L+D \asymp \tau E_{t}$. Consequently $\Delta\left(\begin{array}{cc}A & B \\ 0 & D\end{array}\right)$ is isometric to $\Delta\left(\begin{array}{cc}A & B \\ 0 & \tau E_{t}\end{array}\right)$.

To show that $\tau \in \eta^{-1} \mathcal{P}(k)$ is unique, we compute the Arf invariant of our quadratic form $Q:=\Delta\left(\begin{array}{cc}A & B \\ 0 & \tau E_{t}\end{array}\right)$. Let $S=\left(\begin{array}{cc}0 & B \\ B^{T} & 0\end{array}\right)$ be the polarization of $Q$. Now

$$
\left(\begin{array}{cc}
B^{-1} & 0 \\
0 & I_{n}
\end{array}\right)\left(\begin{array}{cc}
A & B \\
0 & \tau E_{t}
\end{array}\right)\left(\begin{array}{cc}
B^{-T} & 0 \\
0 & I_{n}
\end{array}\right)=\left(\begin{array}{cc}
\tilde{A} & I_{n} \\
0 & \tau E_{t}
\end{array}\right)
$$

is the matrix of $Q$ with respect to a symplectic basis $\left(u_{1}, \ldots, u_{n}, v_{1}, \ldots, v_{n}\right)$ of $M$ relative to $S$. This means that $S\left(u_{i}, u_{j}\right)=S\left(v_{i}, v_{j}\right)=0$ and $S\left(u_{i}, v_{j}\right)=\delta_{i j}$, for all $i, j$. Then the Arf invariant of $Q$ is $\sum_{i=1}^{n} Q\left(u_{i}\right) Q\left(v_{i}\right)=\widetilde{A}_{t, t} \tau=\tau \eta$ (see [4, Theorem 13.13]). It follows that if $\tau^{\prime} \in \eta^{-1} \mathcal{P}(k)$ and $\tau^{\prime} \neq \tau$ then $\Delta\left(\begin{array}{cc}A & B \\ 0 & \tau^{\prime} E_{t}\end{array}\right)$ is not isometric to $\Delta\left(\begin{array}{cc}A & B \\ 0 & \tau E_{t}\end{array}\right)$.

Recall that $\mathcal{D} \mathcal{H}_{n+1 \times n}(k)$ is the set of monic $(n+1) \times n$ Hankel matrices in $\operatorname{sp}\left(H_{2}\right.$, $\left.\left(H_{3}+H_{4}\right),\left(H_{5}+H_{6}\right), \ldots,\left(H_{2 n-1}+H_{2 n}\right), H_{2 n+1}\right)$. 
Theorem 6.7. Given $\alpha \in k^{\times}$and $H \in \mathcal{H}_{(n+1) \times n}(k)$, there is a G-invariant quadratic form on $A_{n} \oplus B_{n}$ which polarizes to $\left(\begin{array}{cc}0 & B \\ B^{T} & 0\end{array}\right)$ with $B=\left(\begin{array}{cc}0 & \alpha \widetilde{I}_{n} \\ \alpha \widetilde{I}_{n+1} & H\end{array}\right)$ if and only if $H \in \mathcal{D H}_{(n+1) \times n}(k)$.

Suppose that $H=\mu_{1} H_{2}+\sum_{i=2}^{n} \mu_{i}\left(H_{2 i-1}+H_{2 i}\right)+\mu_{n+1} H_{2 n+1}$, with $\mu_{1}, \ldots, \mu_{n+1} \in k$.

(i) Set $D=\operatorname{diag}\left(0^{n}, \mu_{1}, \ldots, \mu_{n+1}\right) \in \operatorname{Mat}_{2 n+1}(k)$. Then the G-invariant quadratic forms which polarize to $\left(\begin{array}{cc}0 & B \\ B^{T} & 0\end{array}\right)$ are $\left\{\Delta\left(\begin{array}{cc}D & B \\ 0 & E\end{array}\right) \mid E \in \operatorname{Diag}_{2 n+1}(k)\right\}$.

(ii) If $H=0$ then $\Delta\left(\begin{array}{ll}0 & B \\ 0 & E\end{array}\right)$ is isometric to $\Delta\left(\begin{array}{cc}0 & B \\ 0 & 0\end{array}\right)$.

If $H \neq 0$, there exists $t \in\{1, \ldots, n+1\}$ such that $\mu_{n+2-t} \neq 0$. Then

$$
\operatorname{QIsom}\left(A_{n} \oplus B_{n},\left(\begin{array}{cc}
0 & B \\
B^{T} & 0
\end{array}\right)\right)=\left\{\Delta\left(\begin{array}{cc}
D & B \\
0 & \tau E_{t}
\end{array}\right) \mid \tau \in \mu_{n+2-t}^{-1} \mathcal{P}(k)\right\}
$$

Proof. By (2) the quadratic forms which polarize to $\left(\begin{array}{cc}0 & B \\ B^{T} & 0\end{array}\right)$ are $\Delta Q$, where $Q=\left(\begin{array}{cc}D & B \\ 0 & E\end{array}\right)$, with $D, E \in \operatorname{Diag}_{2 n+1}(k)$. According to (3), $\Delta Q$ is $G$-invariant if and only if $Q-g^{T} Q g$ is alternating, for all $g \in G$. Write $D=\operatorname{diag}\left(d_{1}, \ldots, d_{2 n+1}\right)$ where $d_{1}, \ldots, d_{2 n+1} \in k$.

We described the action of $n_{2}=g_{1}+g_{2}$ on $A_{n} \oplus B_{n}$ in (10). So

$$
\begin{aligned}
g_{2}^{T} Q g_{2} & =\left(\begin{array}{cc}
I_{2 n+1} & 0 \\
T_{2}^{T} & I_{2 n+1}
\end{array}\right) \cdot\left(\begin{array}{cc}
D & B \\
0 & E
\end{array}\right) \cdot\left(\begin{array}{cc}
I_{2 n+1} & T_{2} \\
0 & I_{2 n+1}
\end{array}\right) \\
& =Q+\left(\begin{array}{cc}
0 & D T_{2} \\
T_{2}^{T} D & 0
\end{array}\right)+\left(\begin{array}{cc}
0 & 0 \\
0 & T_{2}^{T} D T_{2}+T_{2}^{T} B
\end{array}\right) .
\end{aligned}
$$

Now $T_{2}^{T} B=B^{T} T_{2}$, and thus $T_{2}^{T} D T_{2}+T_{2}^{T} B$ is symmetric. It follows that $Q-g_{2}^{T} Q g_{2}$ is alternating if and only if $T_{2}^{T} D T_{2}+T_{2}^{T} B$ is hollow. Now $T_{2}^{T}=T_{0}$ and

$$
\left(T_{0} \cdot D \cdot T_{2}+T_{0} \cdot B\right)_{i i}= \begin{cases}0 & \text { if } i=1 \\ d_{i-1}+B_{i-1, i}, & \text { if } i \in\{2, \ldots, 2 n+1\}\end{cases}
$$

So $Q-g_{2}^{T} Q g_{2}$ is alternating if and only if $d_{i}=B_{i, i+1}$, for all $i=1, \ldots, 2 n$.

Similarly $Q-g_{3}^{T} Q g_{3}$ is alternating if and only if $\left(I_{2 n+1}+E_{n+1}\right) D\left(I_{2 n+1}+E_{n+1}\right)+$ $\left(I_{2 n+1}+E_{n+1}\right) B$ is hollow, or equivalently if and only if $d_{i}=B_{i, i}$, for all $i \in\{1, \ldots$, $2 n+1\} \backslash\{n+1\}$.

Write $H=\mu_{1} H_{2}+\sum_{i=2}^{n}\left(\mu_{i}^{\prime} H_{2 i-1}+\mu_{i} H_{2 i}\right)+\mu_{n+1} H_{2 n+1}$, with $\mu_{i}, \mu_{i}^{\prime} \in k$. Then the superdiagonal and diagonal of $B$ are

$$
\left(0^{n}, \mu_{1}, \mu_{2}, \ldots, \mu_{n}\right) \in k^{2 n} \quad \text { and } \quad\left(0^{n}, \alpha, \mu_{2}^{\prime}, \mu_{3}^{\prime}, \ldots, \mu_{n}^{\prime}, \mu_{n+1}\right) \in k^{2 n+1} \quad \text { respectively. }
$$

So by the work above, $\Delta Q$ is $G$-invariant if and only if $\mu_{i}^{\prime}=\mu_{i}$, for $i=2, \ldots, n$ and 


$$
D=\operatorname{diag}\left(0^{n}, \mu_{1}, \ldots, \mu_{n}, \mu_{n+1}\right)
$$

Thus $H=\mu_{1} H_{2}+\sum_{i=2}^{n} \mu_{i}\left(H_{2 i-1}+H_{2 i}\right)+\mu_{n+1} H_{2 n+1}$ belongs to $\mathcal{D} \mathcal{H}_{(n+1) \times n}(k)$. Moreover, when $H \in \mathcal{D} \mathcal{H}_{(n+1) \times n}(k)$ then $D$ is as described in the statement of (i).

We now prove (ii). Let $\left(\begin{array}{cc}D & B \\ 0 & E\end{array}\right)$ with $E \in \operatorname{Diag}_{2 n+1}(k)$ and $D$ as in part (i). Note that all assumptions of Lemma 6.6 are satisfied.

If $H=0$ then $D=0$ and $\Delta\left(\begin{array}{ll}0 & B \\ 0 & E\end{array}\right)$ is isometric to $\Delta\left(\begin{array}{ll}0 & B \\ 0 & 0\end{array}\right)$, by Lemma 6.6(i).

If $H \neq 0$ then $D \neq 0$. Then by direct calculation $B^{-1} D B^{-T}=\widetilde{I}_{2 n+1} D \widetilde{I}_{2 n+1}=$ $\operatorname{diag}\left(\mu_{n+1}, \mu_{n}, \ldots, \mu_{1}, 0^{n}\right)$. Choose $t \in\{1, \ldots, n+1\}$ with $\left(B^{-1} D B^{-T}\right)_{t, t}=\mu_{n+2-t} \neq 0$. Then it follows from Lemma 6.6(ii) that there is a unique $\tau \in \mu_{n+2-t}^{-1} \mathcal{P}(k)$ such that $\Delta\left(\begin{array}{ll}D & B \\ 0 & E\end{array}\right)$ is isometric to $\Delta\left(\begin{array}{cc}D & B \\ 0 & \tau E_{t}\end{array}\right)$. This completes the proof.

\section{The modules $C_{n}(\pi)$ and $C_{n}(\pi)^{2}$}

\subsection{The modules $C_{n}(\pi)$}

Let $\pi \in k[x]$ be a monic irreducible $k$-polynomial of degree $m$ and let $n \geq 1$. Recall the results of Section 3.2 on the companion matrix $\mathcal{P} \in \operatorname{Mat}_{m n}(k)$ of $\pi^{n}$. In particular the Jordan decomposition of $\mathcal{P}$ is

$$
\mathcal{P}=\mathcal{E}+\mathcal{N}
$$

where $\mathcal{E}$ has minimal polynomial $\pi$ and $\mathcal{N}$ is nilpotent, of nilpotency index $n$. Also $K=k[\mathcal{E}]$ is a field extension of $k$ of degree $m$ and $\mathrm{C}(\mathcal{P})=k[\mathcal{P}]$ coincides with the set of $K$-polynomials in $\mathcal{N}$ of degree less than $n$. Finally $\mathrm{T}(\mathcal{P})=\mathcal{T} k[\mathcal{P}]$, where $\mathcal{T} \in \mathrm{GL}_{m n}(k)$ is as defined in $(7)$. In particular $\mathcal{T} \in \mathrm{T}(\mathcal{P})$.

As before, set $n_{2}=g_{1}+g_{2}$ and $n_{3}=g_{1}+g_{3}$ in $k G$. Write $\pi^{n}=\sum_{i=0}^{m n} \lambda_{i} x^{i}$, with $\lambda_{i} \in k$. Following Conlon [1], $C_{n}(\pi)$ is the $2 m n$-dimensional $k G$-module with a basis $\mathcal{B}:=\left(a_{0}, \ldots, a_{m n-1}, b_{1}, \ldots, b_{m n}\right)$ with respect to which the $k G$-action is given by:

$$
\begin{aligned}
& n_{2} a_{j}=n_{3} a_{j}=0, \quad j=0, \ldots, m n-1, \\
& n_{2} b_{j}=a_{j-1}, \quad j=1, \ldots, m n, \\
& n_{3} b_{j}=a_{j}, \quad j=1, \ldots, m n-1, \quad \text { and } \quad n_{3} b_{m n}=\sum_{i=1}^{m n-1} \lambda_{i} a_{i} .
\end{aligned}
$$

So the matrices of $n_{2}$ and $n_{3}$ with respect to $\mathcal{B}$ are

$$
n_{2}=\left(\begin{array}{cc}
0 & I_{m n} \\
0 & 0
\end{array}\right), \quad n_{3}=\left(\begin{array}{cc}
0 & \mathcal{P} \\
0 & 0
\end{array}\right)
$$

Lemma 7.1. $\operatorname{End}_{G}\left(C_{n}(\pi)\right)=\left\{\left(\begin{array}{cc}A & B \\ 0 & A\end{array}\right) \mid A \in \mathrm{C}(\mathcal{P}), B \in \operatorname{Mat}_{m n}(k)\right\}$. 
Proof. Let $A, B, C, D \in \operatorname{Mat}_{m n}(k)$. Then $\left(\begin{array}{cc}A & B \\ C & D\end{array}\right) \in \operatorname{End}_{G}\left(C_{n}(\pi)\right)$ if and only if $A=D$, $C=0$ and $A \mathcal{P}=\mathcal{P} A$.

Lemma 7.2. $\left(\begin{array}{cc}0 & \mathcal{T} \\ \mathcal{T} & 0\end{array}\right)$ is a symplectic form on $C_{n}(\pi)$. In particular $C_{n}(\pi)^{*} \cong C_{n}(\pi)$.

Proof. Set $B=\left(\begin{array}{cc}0 & \mathcal{T} \\ \mathcal{T} & 0\end{array}\right)$. Then $g_{2}{ }^{T} B g_{2}=B$ and $g_{3}{ }^{T} B g_{3}=B$. So $B$ is a non-degenerate $G$-invariant bilinear form on $C_{n}(\pi)$.

Corollary 7.3. $\overline{\operatorname{End}_{G}\left(C_{n}(\pi)\right)} \cong K$ and the adjoint of $\left(\begin{array}{cc}0 & \mathcal{T} \\ \mathcal{T} & 0\end{array}\right)$ acts as the identity on $\overline{\operatorname{End}_{G}\left(C_{n}(\pi)\right)}$.

Proof. Let $\left(\begin{array}{cc}A & B \\ 0 & A\end{array}\right) \in \operatorname{End}_{G}\left(C_{n}(\pi)\right)$, with $A \in \mathrm{C}(\mathcal{P}), B \in \operatorname{Mat}_{m n}(k)$. We may write $A=a(\mathcal{N})$, where $a(x) \in K[x]$. Then $\left(\begin{array}{cc}A & B \\ 0 & A\end{array}\right) \mapsto a\left(0_{k}\right)$ is a $k$-algebra epimorphism onto $K$, with kernel $\mathrm{J}\left(\operatorname{End}_{G}\left(C_{n}(\pi)\right)\right)$. It follows that $\overline{\operatorname{End}_{G}\left(C_{n}(\pi)\right)} \cong K$.

Next let ${ }^{o}$ be the adjoint of $\left(\begin{array}{cc}0 & \mathcal{T} \\ \mathcal{T} & 0\end{array}\right)$ on $\operatorname{End}_{G}\left(C_{n}(\pi)\right)$. Then

$$
\left(\begin{array}{ll}
A & B \\
0 & A
\end{array}\right)^{O}=\left(\begin{array}{cc}
0 & \mathcal{T}^{-1} \\
\mathcal{T}^{-1} & 0
\end{array}\right)\left(\begin{array}{cc}
A^{T} & 0 \\
B^{T} & A^{T}
\end{array}\right)\left(\begin{array}{cc}
0 & \mathcal{T} \\
\mathcal{T} & 0
\end{array}\right)=\left(\begin{array}{cc}
\mathcal{T}^{-1} A^{T} \mathcal{T} & \mathcal{T}^{-1} B^{T} \mathcal{T} \\
0 & \mathcal{T}^{-1} A^{T} \mathcal{T}
\end{array}\right)
$$

But $A^{T} \mathcal{T}=\mathcal{T} A$. So $\mathcal{T}^{-1} A^{T} \mathcal{T}=A$. Hence ${ }^{o}$ acts as the identity on $\overline{\operatorname{End}_{G}\left(C_{n}(\pi)\right)}$.

Lemma 7.4. $\left\{\left(\begin{array}{cc}0 & B \\ B & D\end{array}\right) \mid B \in \mathrm{T}(\mathcal{P}), D \in \operatorname{Mat}_{m n}(k)\right\}$ are the $G$-invariant bilinear forms on $C_{n}(\pi)$. A form is symplectic if and only if $B$ is invertible and $D$ is alternating.

Proof. Let $A \in \mathrm{C}(\mathcal{P})$ and $X \in \operatorname{Mat}_{m n}(k)$. Then $\left(\begin{array}{cc}0 & \mathcal{T} \\ \mathcal{T} & 0\end{array}\right)\left(\begin{array}{cc}A & X \\ 0 & A\end{array}\right)=\left(\begin{array}{cc}0 & \mathcal{T} A \\ \mathcal{T} A & \mathcal{T} X\end{array}\right)$. The result now follows from Lemmas 7.1 and 7.2 .

Theorem 7.5. $\operatorname{Isom}\left(C_{n}(\pi)\right)=\left\{\left(\begin{array}{cc}0 & \mathcal{T}\left(I_{m n}+\mathcal{N} f^{2}\right) \\ \mathcal{T}\left(I_{m n}+\mathcal{N} f^{2}\right) & 0\end{array}\right) \mid \begin{array}{c}f \in K[\mathcal{N}] \\ \operatorname{deg}(f) \leq \frac{n-2}{2}\end{array}\right\}$.

Proof. Let $B \in \mathrm{T}(\mathcal{P})$ be invertible and let $D \in \operatorname{Mat}_{m n}(k)$ be alternating. So $\left(\begin{array}{ll}0 & B \\ B & D\end{array}\right)$ is an indecomposable symplectic form on $C_{n}(\pi)$. Let $X$ be a unit in $\mathrm{C}(\mathcal{P})$ and let $Y \in$ $\operatorname{Mat}_{m n}(k)$. Then $\left(\begin{array}{cc}X & Y \\ 0 & X\end{array}\right)$ is a unit in $\operatorname{End}_{G}\left(C_{n}(\pi)\right)$ and

$$
\left(\begin{array}{cc}
X & Y \\
0 & X
\end{array}\right)^{T}\left(\begin{array}{cc}
0 & B \\
B & D
\end{array}\right)\left(\begin{array}{cc}
X & Y \\
0 & X
\end{array}\right)=\left(\begin{array}{cc}
0 & X^{T} B X \\
X^{T} B X & Y^{T} B X+X^{T} B Y+X^{T} D X
\end{array}\right)
$$

Note that as $D$ is alternating, there is some $Z \in \operatorname{Mat}_{m n}(k)$ so that $D=Z+Z^{T}$. Choosing $X=I_{m n}$ and $Y=B^{-1} Z$ we see that $\left(\begin{array}{cc}0 & B \\ B & D\end{array}\right)$ is isometric to $\left(\begin{array}{cc}0 & B \\ B & 0\end{array}\right)$. Furthermore $\left(\begin{array}{ll}0 & B \\ B & 0\end{array}\right)$ is isometric to some $\left(\begin{array}{cc}0 & C \\ C & 0\end{array}\right)$ if and only if $C=X^{T} B X$, for some unit $X \in \mathrm{C}(\mathcal{P})$. But $X^{T} B X=B X^{2}$. Now by Lemma 3.4 there are $X, Y \in \mathrm{C}(\mathcal{P})$ such that $\mathcal{T}^{-1} B X^{2}=$ $I_{m n}+\mathcal{N} Y^{2}$. Moreover $I_{m n}+\mathcal{N} Y^{2}$ is uniquely determined. Clearly we may choose $Y=f$, 
where $f \in K[\mathcal{N}]$ is a unique $K$-polynomial in $\mathcal{N}$ of degree at most $(n-2) / 2$. This completes the proof.

Corollary 7.6. If $k=\mathbb{F}_{q}$ then $\left|\operatorname{Isom}\left(C_{n}(\pi)\right)\right|=q^{m\left\lfloor\frac{n}{2}\right\rfloor}$.

Recall that $\mathcal{P}(k)$ is a set of representatives for the cosets of $\left\{\lambda^{2}+\lambda \mid \lambda \in k\right\}$ in $(k,+)$.

Theorem 7.7. For a unit $B \in \mathrm{T}(\mathcal{P})$, there is a $G$-invariant quadratic form on $C_{n}(\pi)$ which polarizes to $\left(\begin{array}{cc}0 & B \\ B & 0\end{array}\right)$ if and only if one of the following holds:

(i) $\pi \in\{x, x+1\}$, $n$ is odd and $\left(\begin{array}{cc}0 & B \\ B & 0\end{array}\right)$ is isometric to $\left(\begin{array}{cc}0 & \mathcal{T}\left(I_{m n}+\mathcal{N}\right) \\ \mathcal{T}\left(I_{m n}+\mathcal{N}\right) & 0\end{array}\right)$.

(ii) $\pi \notin\{x, x+1\}$, $n$ is even and $\left(\begin{array}{cc}0 & B \\ B & 0\end{array}\right)$ is isometric to $\left(\begin{array}{cc}0 & \mathcal{T}\left(\mathcal{P}+\mathcal{P}^{2}\right) \\ \mathcal{T}\left(\mathcal{P}+\mathcal{P}^{2}\right) & 0\end{array}\right)$.

If (i) or (ii) holds, let $D \in \operatorname{Diag}_{m n}(k)$ be the diagonal of $B$. Then the corresponding $G$-invariant quadratic forms on $C_{n}(\pi)$ are $\left\{\Delta\left(\begin{array}{cc}D & B \\ 0 & E\end{array}\right) \mid E \in \operatorname{Diag}_{m n}(k)\right\}$. Moreover, we can choose $t \in\{1, \ldots, m n\}$ such that $\left(B^{-1}\right)_{t, t} \neq 0_{k}$. For any such $t, \Delta\left(\begin{array}{cc}D & B \\ 0 & E\end{array}\right)$ is isometric to a unique $\Delta\left(\begin{array}{cc}D & B \\ 0 & \tau E_{t}\end{array}\right)$ with $\tau \in\left(B^{-1}\right)_{t, t}^{-1} \mathcal{P}(k)$.

Proof. By (2) a quadratic form with polarizes to $\left(\begin{array}{cc}0 & B \\ B & 0\end{array}\right)$ is given by $\Delta Q$, where $Q=$ $\left(\begin{array}{cc}D & B \\ 0 & E\end{array}\right)$ with $D, E \in \operatorname{Diag}_{m n}(k)$. By (3), $\Delta Q$ is $G$-invariant if and only if $Q-g_{j}^{T} Q g_{j}$ is alternating, for $j=2,3$. One checks quickly that

$$
Q-g_{2}^{T} Q g_{2}=\left(\begin{array}{cc}
0 & D \\
D & D+B
\end{array}\right), \quad Q-g_{3}^{T} Q g_{3}=\left(\begin{array}{cc}
0 & D \mathcal{P} \\
\mathcal{P}^{T} D & \mathcal{P}^{T} D \mathcal{P}+\mathcal{P}^{T} B
\end{array}\right)
$$

Notice that $D+B$ and $\mathcal{P}^{T} D \mathcal{P}+\mathcal{P}^{T} B$ are symmetric. Also $\mathcal{P}^{T} D \mathcal{P}+\mathcal{P}^{T} B=\mathcal{P}^{T}(D+$ $B) \mathcal{P}+\left(\mathcal{P}^{T} B \mathcal{P}+\mathcal{P}^{T} B\right)$. So if $D+B$ is hollow, Lemma 3.2 implies that $\mathcal{P}^{T} D \mathcal{P}+\mathcal{P}^{T} B$ is hollow if and only if $\mathcal{P}^{T} B \mathcal{P}+\mathcal{P}^{T} B$ is hollow. Moreover $\mathcal{P}^{T} B \mathcal{P}+\mathcal{P}^{T} B=B\left(\mathcal{P}^{2}+\mathcal{P}\right)$. We deduce that $\Delta Q$ is $G$-invariant if and only if $D+B$ and $B\left(\mathcal{P}^{2}+\mathcal{P}\right)$ are hollow.

First note that $D+B$ is hollow if and only if $D=\operatorname{diag}\left(B_{1,1}, \ldots, B_{m n, m n}\right)$. In particular $D$ is determined by $B$. Next write $B=\mathcal{T} U$, where $U$ is a unit in $\mathrm{C}(\mathcal{P})$.

For (i), suppose that $\pi \in\{x, x+1\}$. Then $\mathcal{P}+\mathcal{P}^{2}=\mathcal{N}+\mathcal{N}^{2}$. If $n$ is even, Corollary 3.7 implies that $B\left(\mathcal{P}^{2}+\mathcal{P}\right)$ is hollow if and only if $U\left(\mathcal{N}+\mathcal{N}^{2}\right)=C^{2}$, for some $C \in \mathrm{C}(\mathcal{P})$. This is impossible, as $\left|U\left(\mathcal{N}+\mathcal{N}^{2}\right)\right|=1$ but $\left|C^{2}\right|$ is even. Here $|\cdot|$ is the valuation given by (5). If $n$ is odd, then Corollary 3.7 implies that $B\left(\mathcal{P}^{2}+\mathcal{P}\right)$ is hollow if and only if $U\left(\mathcal{N}+\mathcal{N}^{2}\right)=\mathcal{N} C^{2}$, for some unit $C \in \mathrm{C}(\mathcal{P})$. This means that $U\left(I_{m n}+\mathcal{N}\right)=$ $C^{2}+\lambda \mathcal{N}^{n-1}$, for some $\lambda \in K$. But $K$ is perfect and $n-1$ is even. So $U X^{2}=\left(I_{m n}+\mathcal{N}\right)$, 
where $X=\left(I_{m n}+\mathcal{N}\right)\left(C+\sqrt{\bar{\lambda}} \mathcal{N}^{(n-1) / 2}\right)^{-1}$ is unit in $\mathrm{C}(\mathcal{P})$. Now the isometry follows from (15) with $Y=0$.

For (ii), suppose that $\pi \notin\{x, x+1\}$. Then $\mathcal{P}+\mathcal{P}^{2}=\left(\mathcal{E}+\mathcal{E}^{2}\right)+\mathcal{N}+\mathcal{N}^{2}$ is a unit in $K[\mathcal{N}]$, as $\mathcal{E} \neq \mathcal{E}^{2}$. If $n$ is odd, Corollary 3.7 implies that $B\left(\mathcal{P}^{2}+\mathcal{P}\right)$ is hollow if and only if $U\left(\mathcal{P}+\mathcal{P}^{2}\right)=\mathcal{N} C^{2}$, for some $C \in \mathrm{C}(\mathcal{P})$. This is impossible, as $\left|U\left(\mathcal{P}+\mathcal{P}^{2}\right)\right|=0$ and $\left|\mathcal{N} C^{2}\right|>0$. If $n$ is even. So Corollary 3.7 implies that $B\left(\mathcal{P}^{2}+\mathcal{P}\right)$ is hollow if and only if $U\left(\mathcal{P}+\mathcal{P}^{2}\right)=C^{2}$, for some unit $C \in \mathrm{C}(\mathcal{P})$. Thus $U X^{2}=\mathcal{P}+\mathcal{P}^{2}$, where $X=\left(\mathcal{P}+\mathcal{P}^{2}\right) C^{-1}$. Again the stated isometry follows from (15) with $Y=0$.

Now suppose that (i) or (ii) hold and let $\Delta\left(\begin{array}{cc}D & B \\ 0 & E\end{array}\right)$ be a $G$-invariant quadratic form on $C_{n}(\pi)$. We claim that $B$ is not hollow. If $n$ is odd this follows at once from Corollary 3.7, as in that case $\mathrm{T}(\mathcal{P})$ contains no hollow units. If $n$ is even then $\pi \notin\{x, x+1\}$ and we may assume that $\mathcal{T}^{-1} B=\left(\mathcal{E}+\mathcal{E}^{2}\right)+\mathcal{N}+\mathcal{N}^{2}$. As this is not a square in $\mathrm{C}(\pi)$, Corollary 3.7 again implies that $B$ is not hollow. This proves our claim.

As $B$ is not hollow, neither is $B^{-1}=B^{-1} B B^{-T}$. Choose $t \in\{1, \ldots, m n\}$ such that $\left(B^{-1}\right)_{t, t} \neq 0$ and set $\nu=\left(B^{-1}\right)_{t, t}$. Now notice that $B^{-1}(D+B) B^{-T}$ is hollow. So $\nu=\left(B^{-1} D B^{-T}\right)_{t, t}$. It now follows from Lemma 6.6(ii) that $\Delta\left(\begin{array}{ll}D & B \\ 0 & E\end{array}\right)$ is isometric to $\Delta\left(\begin{array}{cc}D & B \\ 0 & \tau E_{t}\end{array}\right)$, for a unique $\tau \in \nu^{-1} \mathcal{P}(k)$.

\subsection{The modules $C_{n}(\pi)^{2}$}

Recall the basis $\mathcal{B}:=\left(a_{0}, \ldots, a_{m n-1}, b_{1}, \ldots, b_{m n}\right)$ of $C_{n}(\pi)$. Now $C_{n}(\pi)^{2}$ is the Cartesian product of $C_{n}(\pi)$ with itself. We use the following ordered basis of $C_{n}(\pi)^{2}$ :

$$
\mathcal{C}:=\left(\left(a_{u}, 0\right),\left(0, a_{v}\right),\left(b_{w}, 0\right),\left(0, b_{x}\right)\right), \quad 0 \leq u, v \leq m n-1, \quad 1 \leq w, x \leq m n
$$

Lemma 7.1 can be used to show that, with respect to $\mathcal{C}$

$$
\operatorname{End}_{G}\left(C_{n}(\pi)^{2}\right)=\left\{\left(\begin{array}{cc}
A & B \\
0 & A
\end{array}\right) \mid A \in \operatorname{Mat}_{2}(\mathrm{C}(\mathcal{P})), B \in \operatorname{Mat}_{2 m n}(k)\right\}
$$

In turn Lemmas 2.2 and 7.4 show that the $G$-invariant symplectic forms on $C_{n}(\pi)^{2}$ are

$$
\left\{\left(\begin{array}{cc}
0 & B \\
B & D
\end{array}\right) \mid \begin{array}{ll}
D \in \operatorname{Mat}_{2 m n}(k) \\
\text { alternating }
\end{array}, B=\left(\begin{array}{cc}
\widetilde{B}_{1} & \widetilde{B}_{2} \\
\widetilde{B}_{2} & \widetilde{B}_{4}
\end{array}\right), \begin{array}{c}
\widetilde{B}_{2}, \widetilde{B}_{4} \in \mathrm{T}(\mathcal{P}) \\
\widetilde{B}_{2} \text { nonsingular } \\
\widetilde{B}_{1}, \widetilde{B}_{4} \text { singular }
\end{array}\right\}
$$

Theorem 7.8. Each indecomposable symplectic form on $C_{n}(\pi)^{2}$ is isometric to exactly one form $\left(\begin{array}{cc}0 & B \\ B & 0\end{array}\right)$ with $B=\left(\begin{array}{cc}\mathcal{T} \mathcal{N}^{v}\left(I+\mathcal{N} f_{1}^{2}\right) & \mathcal{T} f_{2} \\ \mathcal{T} f_{2} & \mathcal{T} \mathcal{N}^{w}\end{array}\right)$

Here $0<v<w \leq n+1, w-v$ is odd and $f_{1}, f_{2} \in K[\mathcal{N}]$. Also $f_{1}=0$, if $w=v+1$, and otherwise $\operatorname{deg}\left(f_{1}\right) \leq \frac{w-v-3}{2}$. Moreover if $w \in\{n, n+1\}$ then $f_{2}=I_{m n}$, and otherwise $\operatorname{deg}\left(f_{2}\right) \leq \frac{n-w-1}{2}$ and $f_{2}(0) \neq 0$. 
Proof. Let $\left(\begin{array}{cc}0 & B \\ B & D\end{array}\right)$ be a symplectic form on $C_{n}(\pi)^{2}$. So $D \in \operatorname{Mat}_{2 m n}(k)$ is alternating and $B=\left(\begin{array}{cc}\widetilde{B}_{1} & \widetilde{B}_{2} \\ \widetilde{B}_{2} & \widetilde{B}_{4}\end{array}\right)$ with $\widetilde{B}_{1}, \widetilde{B}_{2}, \widetilde{B}_{4} \in \mathrm{T}(\mathcal{P})$ such that $\widetilde{B}_{2}$ is invertible and $\widetilde{B}_{1}, \widetilde{B}_{4}$ are not invertible. We first show that the isometry class of $\left(\begin{array}{ll}0 & B \\ B & D\end{array}\right)$ contains a form which has one of the given types. We then show that this form is unique in its isometry class.

Let $\left(\begin{array}{cc}X & Y \\ 0 & X\end{array}\right)$ be a unit in $\operatorname{End}_{G}\left(C_{n}(\pi)^{2}\right)$. So $X$ is a unit in $\operatorname{Mat}_{2}(\mathrm{C}(\mathcal{P}))$ and $Y \in$ $\operatorname{Mat}_{2 m n}(k)$. Also

$$
\left(\begin{array}{cc}
X & Y \\
0 & X
\end{array}\right)^{T}\left(\begin{array}{cc}
0 & B \\
B & D
\end{array}\right)\left(\begin{array}{cc}
X & Y \\
0 & X
\end{array}\right)=\left(\begin{array}{cc}
0 & X^{T} B X \\
X^{T} B X & Y^{T} B X+X^{T} B Y+X^{T} D X
\end{array}\right)
$$

Now as $D$ is alternating, there is some $Z \in \operatorname{Mat}_{2 m n}(k)$ such that $D=Z+Z^{T}$. Choosing $X=I_{2 m n}$ and $Y=B^{-1} Z$ we see that $\left(\begin{array}{cc}0 & B \\ B & D\end{array}\right)$ is isometric to $\left(\begin{array}{cc}0 & B \\ B & 0\end{array}\right)$. Moreover $\left(\begin{array}{cc}0 & B \\ B & 0\end{array}\right)$ is isometric to all $\left(\begin{array}{cc}0 & X^{T} B X \\ X^{T} B X & 0\end{array}\right)$ as $X$ ranges over the units of $\operatorname{Mat}_{2}(\mathrm{C}(\mathcal{P}))$.

We consider $X^{T} B X$ in more detail. Write $X=\left(\begin{array}{ll}P & Q \\ R & S\end{array}\right)$, where $P, Q, R, S \in \mathrm{C}(\mathcal{P})$, and $P S+Q R$ is a unit in $\mathrm{C}(\mathcal{P})$. Now $A^{T} \mathcal{T}=\mathcal{T} A$, for all $A \in \mathrm{C}(\mathcal{P})$. Moreover $\mathrm{C}(\mathcal{P})$ is a commutative ring. An easy calculation then gives

$$
X^{T} B X=\left(\begin{array}{cc}
\widetilde{B}_{1} P^{2}+\widetilde{B}_{4} R^{2} & \widetilde{B}_{2}(P S+Q R)+\widetilde{B}_{1} P Q+\widetilde{B}_{4} R S \\
\widetilde{B}_{2}(P S+Q R)+\widetilde{B}_{1} P Q+\widetilde{B}_{4} R S & \widetilde{B}_{1} Q^{2}+\widetilde{B}_{4} S^{2}
\end{array}\right)
$$

So the isometry class of $\left(\begin{array}{cc}0 & B \\ B & D\end{array}\right)$ contains all $\left(\begin{array}{cc}0 & B^{\prime} \\ B^{\prime} & 0\end{array}\right)$ where $B^{\prime}=\left(\begin{array}{cc}\widetilde{B}_{1}^{\prime} & \widetilde{B}_{2}^{\prime} \\ \widetilde{B}_{2}^{\prime} & \widetilde{B}_{4}^{\prime}\end{array}\right)$, and if we write $\widetilde{B}_{i}=\mathcal{T} B_{i}$ and $\widetilde{B}_{i}^{\prime}=\mathcal{T} B_{i}^{\prime}$, with $B_{i}, B_{i}^{\prime} \in \mathrm{C}(\mathcal{P})$, for $i=1,2,4$, then

$$
\begin{aligned}
& B_{1}^{\prime}=B_{1} P^{2}+B_{4} R^{2}, \\
& B_{2}^{\prime}=B_{2}(P S+Q R)+B_{1} P Q+B_{4} R S, \\
& B_{4}^{\prime}=B_{1} Q^{2}+B_{4} S^{2}
\end{aligned}
$$

for some $P, Q, R, S \in \mathrm{C}(\mathcal{P})$ such that $P S+Q R$ is a unit in $\mathrm{C}(\mathcal{P})$.

We consider a number of cases. Set $v=\left|B_{1}\right|$, where $|\cdot|$ is the valuation given by (5). Then $0<v \leq n$, as $B_{1}$ is not invertible in $\mathrm{C}(\mathcal{P})$.

Case 1: Suppose that $B_{1}=B_{4}=0$. Setting $P=B_{2}^{-1}, Q=R=0$ and $S=I_{m n},(16)$ gives $B_{1}^{\prime}=B_{4}^{\prime}=0$ and $B_{2}^{\prime}=I_{m n}$. So $\left(\begin{array}{cc}0 & B \\ B & D\end{array}\right)$ is isometric to $\left(\begin{array}{cc}0 & B^{\prime} \\ B^{\prime} & 0\end{array}\right)$ with $B^{\prime}=\left(\begin{array}{cc}0 & \mathcal{T} \\ \mathcal{T} & 0\end{array}\right)$, and $\left(C_{n}(\pi)^{2},\left(\begin{array}{cc}0 & B \\ B & D\end{array}\right)\right)$ is isometric to the paired module. In this case $v=n, w=n+1$, $f_{1}=0$ and $f_{2}=1$.

Case 2: Suppose that exactly one of $B_{1}$ and $B_{4}$ is 0 . Taking $P=S=0$ and $Q=$ $R=I_{m n}$, (16) gives $B_{1}^{\prime}=B_{4}, B_{2}^{\prime}=B_{2}$ and $B_{4}^{\prime}=B_{1}$. So we may assume, and we do, that $B_{1} \neq 0$ and $B_{4}=0$. Now by Lemma 3.4 there are $V, W \in \mathrm{C}(\mathcal{P})$, with $V$ a unit, such that $B_{1} V^{2}=\mathcal{N}^{v}+\mathcal{N}^{v+1} W^{2}$. Choosing $P=V, Q=R=0$ and $S=V^{-1} B_{2}^{-1}$, (16) gives $B_{1}^{\prime}=\mathcal{N}^{v}+\mathcal{N}^{v+1} W^{2}, B_{2}^{\prime}=I_{m n}$ and $B_{4}^{\prime}=0$. So $\left(\begin{array}{cc}0 & B \\ B & D\end{array}\right)$ is isometric to $\left(\begin{array}{cc}0 & B^{\prime} \\ B^{\prime} & 0\end{array}\right)$ with 
$B^{\prime}=\left(\begin{array}{cc}\mathcal{T}\left(\mathcal{N}^{v}+\mathcal{N}^{v+1} W^{2}\right) & \mathcal{T} \\ 0\end{array}\right)$. We note that $\mathcal{T} \mathcal{N}^{v+1} W^{2}=\mathcal{T} \mathcal{N}^{v+1} f_{1}^{2}$, for some $f_{1} \in K[\mathcal{N}]$ such that $v+1+2 \operatorname{deg}\left(f_{1}\right) \leq n-1$. Choose $w \in\{n, n+1\}$ such that $w-v$ is odd. Then $\operatorname{deg}\left(f_{1}\right) \leq(w-v-3) / 2$. Also in this case $f_{2}=I_{m n}$.

Final Case: We now suppose that $B_{1} \neq 0$ and $B_{4} \neq 0$. Also set $w=\left|B_{4}\right|$. We may assume that $v \leq w$ and that $w-v$ is maximal in the isometry class of $\left(\begin{array}{ll}0 & B \\ B & D\end{array}\right)$.

We claim that $w-v$ is odd. For suppose otherwise. Then take $P=I_{m n}, Q=$ $\lambda \mathcal{N}^{(w-v) / 2}$, for some $\lambda \in K, R=0$ and $S=I_{m n}$. Then (16) gives $B_{1}^{\prime}=B_{1}, B_{4}^{\prime}=$ $B_{1} \lambda^{2} \mathcal{N}^{(w-v)}+B_{4}$. But $\left|B_{1} \lambda^{2} \mathcal{N}^{(w-v)}\right|=\left|B_{4}\right|$. So we may choose $\lambda$ so that $\left|B_{4}^{\prime}\right|>\left|B_{4}\right|$. Then $\left|B_{4}^{\prime}\right|-\left|B_{1}^{\prime}\right|>w-v$, which contradicts the maximality of $w-v$. This proves our claim.

Now by Lemma 3.4 there are $V_{1}, W_{1} \in \mathrm{C}(\mathcal{P})$, with $V_{1}$ a unit, such that $B_{1} V_{1}^{2}=$ $\mathcal{N}^{v}+\mathcal{N}^{v+1} W_{1}^{2}$. Choosing $P=V_{1}, Q=R=0$ and $S=I_{m n}$ in (16), we can replace $B_{1}$ by $B_{1}^{\prime}=\mathcal{N}^{v}+\mathcal{N}^{v+1} W_{1}^{2}$ and $B_{4}^{\prime}=B_{4}$. By similar reasoning, we may assume that $B_{4}=\mathcal{N}^{w}+\mathcal{N}^{w+1} W_{4}^{2}$, for some $W_{4} \in \mathrm{C}(\mathcal{P})$.

Next take $P=I_{m n}, Q=\mathcal{N}^{(w-v+1) / 2} W_{4}, R=0, S=I_{m n}$. Then $B_{1}^{\prime}=B_{1}$ and we may replace $B_{4}$ by $B_{4}^{\prime}=\mathcal{N}^{w}+\mathcal{N}^{w+2}\left(W_{1} W_{4}\right)^{2}$. Now choose $P=I_{m n}, Q=R=0, S=$ $\left(I_{m n}+\mathcal{N} W_{1} W_{4}\right)^{-1}$. Then $B_{1}^{\prime}=B_{1}$ and we can replace $B_{4}$ by $B_{4}^{\prime}=\mathcal{N}^{w}$.

Write $B_{1}=\mathcal{N}^{v}+\mathcal{N}^{v+1} f_{1}\left(\mathcal{N}^{2}\right)+\mathcal{N}^{w} L^{2}$, where $f_{1} \in K[x]$ and $\operatorname{deg}\left(f_{1}\right) \leq(w-v-3) / 2$ and $L \in \mathrm{C}(\mathcal{P})$. Choosing $P=I_{m n}, Q=0, R=L, S=I_{m n}$, we get $B_{1}^{\prime}=\mathcal{N}^{v}+$ $\mathcal{N}^{v+1} f_{1}\left(\mathcal{N}^{2}\right)$ and $B_{4}^{\prime}=B_{4}$.

We now modify $B_{2}$ as much as we can, without changing $B_{1}$ or $B_{4}$. Notice that the annihilator of $\mathcal{N}^{w}$ in $K[\mathcal{N}]$ is generated by $\mathcal{N}^{n-w}$. So $t:=\left\lfloor\frac{n-w-1}{2}\right\rfloor$ is the largest integer such that $w+2 t<n$. Next write $B_{2}=f_{2}(\mathcal{N})+E \mathcal{N}^{t+1}$, where $f_{2} \in K[x]$ with $\operatorname{deg}\left(f_{2}\right) \leq t$ and $E \in \mathrm{C}(\mathcal{P})$. Choosing $P=I_{m n}, Q=R=0, S=\left(1+B_{2}^{-1} E \mathcal{N}^{t+1}\right)$ we get $B_{1}^{\prime}=B_{1}$, $B_{4}^{\prime}=B_{4}$, and we replace $B_{2}$ by $B_{2}^{\prime}=B_{2}+E \mathcal{N}^{t+1}=f_{2}(\mathcal{N})$.

Uniqueness: Let $\left(\begin{array}{cc}0 & B \\ B & 0\end{array}\right)$ and $\left(\begin{array}{cc}0 & B^{\prime} \\ B^{\prime} & 0\end{array}\right)$ be isometric symplectic forms on $C_{n}(\pi)^{2}$ with

$$
\begin{aligned}
& B_{1}=\mathcal{N}^{v}+\mathcal{N}^{v+1} f_{1}\left(\mathcal{N}^{2}\right), \quad B_{2}=f_{2}(\mathcal{N}), \quad B_{4}=\mathcal{N}^{w}, \\
& B_{1}^{\prime}=\mathcal{N}^{x}+\mathcal{N}^{x+1} f_{1}^{\prime}\left(\mathcal{N}^{2}\right), \quad B_{2}^{\prime}=f_{2}^{\prime}(\mathcal{N}), \quad B_{4}^{\prime}=\mathcal{N}^{y},
\end{aligned}
$$

where $v, w, f_{1}, f_{2}$, and likewise $x, y, f_{1}^{\prime}, f_{2}^{\prime}$, satisfy the constraints in the statement of the theorem. Moreover (16) holds for certain $P, Q, R, S \in \mathrm{C}(\mathcal{P})$.

First note that (16) implies that $\left|B_{1}^{\prime}\right| \geq \min \left\{\left|B_{1}\right|+2|P|,\left|B_{4}\right|+2|R|\right\} \geq\left|B_{1}\right|$. But as isometry is a symmetric relation, we likewise have $\left|B_{1}\right| \geq\left|B_{1}^{\prime}\right|$. So $v=x$.

We claim that $B_{4}^{\prime}=B_{4}$. For otherwise we may assume that $B_{4}^{\prime} \neq 0$ and $\left|B_{4}\right|>\left|B_{4}^{\prime}\right|$. Now $\left|B_{1} Q^{2}\right| \neq\left|B_{4} S^{2}\right|$. So $\left|B_{4}^{\prime}\right|=\min \left\{\left|B_{1}\right|+2|Q|,\left|B_{4}\right|+2|S|\right\}$. This forces $\left|B_{4}^{\prime}\right|=$ $\left|B_{1}\right|+2|Q|=\left|B_{1}^{\prime}\right|+2|Q|$. So $y-x$ is even, which is false. This proves our claim.

The equation for $B_{4}^{\prime}$ in (16) can now be rearranged to

$$
\mathcal{N}^{w}\left(I_{m n}+S^{2}\right)=B_{1} Q^{2}
$$


If $B_{1} Q^{2} \neq 0$, then $\left|I_{m n}+S^{2}\right|$ is even. So $w \equiv v \bmod 2$, which is false. So in fact $B_{1} Q^{2}=0$ and $\mathcal{N}^{w}\left(I_{m n}+S^{2}\right)=0$. Recall that $t$ is the largest integer such that $2 t<(n-w)$. Then

$$
Q \equiv 0 \bmod \mathcal{N}^{t+1}, \quad \text { and } \quad S \equiv I_{m n} \quad \bmod \mathcal{N}^{t+1}
$$

The equation for $B_{1}^{\prime}$ in (16) can likewise be rearranged to

$$
\mathcal{N}^{v}\left(I_{m n}+P^{2}\right)=\mathcal{N}^{v+1}\left(f_{1}^{\prime}\left(\mathcal{N}^{2}\right)+f_{1}\left(\mathcal{N}^{2}\right) P^{2}\right)+\mathcal{N}^{w} R^{2}
$$

As $w-v$ is odd, it follows that both sides are 0 . So $\mathcal{N}^{v}=\mathcal{N}^{v} P^{2}$. Substituting this back into the previous displayed equation gives

$$
\mathcal{N}^{v+1}\left(f_{1}^{\prime}+f_{1}\right)\left(\mathcal{N}^{2}\right)=\mathcal{N}^{w} R^{2}
$$

But $\operatorname{deg}\left(f_{1}^{\prime}+f_{1}\right) \leq \frac{w-v-3}{2}$. So $\mathcal{N}^{w} R^{2}=0$ and $f_{1}^{\prime}\left(\mathcal{N}^{2}\right)=f_{1}\left(\mathcal{N}^{2}\right)$, whence $B_{1}^{\prime}=B_{1}$.

Now $\mathcal{N}^{v}=\mathcal{N}^{v} P^{2}$ and $\mathcal{N}^{w} R^{2}=0$ implies that

$$
\text { and } \quad P \equiv I_{m n} \quad \bmod \mathcal{N}^{t+1}, \quad \text { and } \quad R \equiv 0 \quad \bmod \mathcal{N}^{t+1}
$$

Finally, we may use (17), (18) and the equation for $B_{2}^{\prime}$ in (16) to obtain the congruence

$$
\left(f_{2}+f_{2}^{\prime}\right)(\mathcal{N}) \equiv 0 \quad \bmod \mathcal{N}^{t+1}
$$

But $\operatorname{deg}\left(f_{2}+f_{2}^{\prime}\right) \leq t$. So $B_{2}=f_{2}(\mathcal{N})=f_{2}^{\prime}(\mathcal{N})=B_{2}^{\prime}$.

Corollary 7.9. If $k$ is finite, set $q:=|k|^{m}$. Then

$$
\left|\operatorname{Isom}\left(C_{n}(\pi)^{2}\right)\right|= \begin{cases}n \cdot q^{\frac{n-2}{2}}, & \text { if } n \text { is even } \\ \left(\frac{n+1}{2} q+\frac{n-1}{2}\right) \cdot q^{\frac{n-3}{2}}, & \text { if } n \text { is odd }\end{cases}
$$

Proof. We count the number of choices we have for $v, w$ and $f_{1}, f_{2} \in K[\mathcal{N}]$, subject to the constraints of Theorem 7.8. So fix $v$, with $0<v \leq n$. The case $v=n$ corresponds to $B_{1}=B_{4}=0$ and $f_{2}=I_{m n}$. So there is one such isometry class.

From now on we assume that $v<n$. Set $\ell:=\left\lfloor\frac{n-v}{2}\right\rfloor$. So $\ell=\mid\{w \mid v<w<n$, $w-v$ odd $\} \mid$. First note that $w \in\{n, n+1\}$ corresponds to $B_{4}=0, f_{2}=I_{m n}$ and $\operatorname{deg}\left(f_{1}\right) \leq \frac{w-v-2}{2}$. So we have $q^{\ell}$ choices for $f_{1}$. Suppose then that $w<n$. Then $\operatorname{deg}\left(f_{1}\right) \leq$ $\frac{w-v-3}{2}, \operatorname{deg}\left(f_{2}\right) \leq \frac{n-w-1}{2}$ and $f_{2}(0) \neq 0$ as $f_{2}$ is a unit. So there are $q^{(w-v-1) / 2}$ choices for $f_{1}$ and $(q-1) q^{\left\lfloor\frac{\left\lfloor^{2}-w-1\right.}{2}\right\rfloor}$ choices for $f_{2}$. But $\ell=\left\lfloor\frac{n-w-1}{2}\right\rfloor+\frac{w-v+1}{2}$ as $w-v \equiv 1$ $\bmod 2$. So the number of choices for the pair $f_{1}, f_{2}$ is $(q-1) q^{\ell-1}$. Now as there are $\ell$ allowed values of $w$ strictly between $v$ and $n$, the total number of isometry classes with parameter $v<n$ is

$$
q^{\ell}+\ell(q-1) q^{\ell-1}=(\ell+1) q^{\ell}-\ell q^{\ell-1}
$$


Suppose that $n$ is even. Then as $v$ ranges over $\{1, \ldots, n\}, \ell$ takes each value in $\left\{0, \ldots, \frac{n-2}{2}\right\}$ twice. So the total number of isometry classes of symplectic forms is given by the telescoping sum

$$
2 \sum_{\ell=0}^{\frac{n-2}{2}}\left((\ell+1) q^{\ell}-\ell q^{\ell-1}\right)=n q^{\frac{n-2}{2}}
$$

Suppose that $n$ is odd. Then as $v$ ranges over $\{1, \ldots, n\}, \ell$ takes each value in $\left\{0, \ldots, \frac{n-3}{2}\right\}$ twice and $\frac{n-1}{2}$ once. So now the total number of isometry classes of symplectic forms is given by the sum

$$
2 \sum_{\ell=0}^{\frac{n-3}{2}}\left((\ell+1) q^{\ell}-\ell q^{\ell-1}\right)+\left(\frac{n+1}{2} q^{\frac{n-1}{2}}-\frac{n-1}{2} q^{\frac{n-3}{2}}\right)=\frac{n+1}{2} q^{\frac{n-1}{2}}+\frac{n-1}{2} q^{\frac{n-3}{2}}
$$

Theorem 7.10. There is a G-invariant quadratic form on $C_{n}(\pi)^{2}$ which polarizes to $\left(\begin{array}{cc}0 & B \\ B & 0\end{array}\right)$ if and only if there is a unit $U \in \operatorname{Mat}_{2}(\mathrm{C}(\mathcal{P}))$ and one of the following holds:

(i) $U^{T} B U=\left(\begin{array}{cc}0 & \mathcal{T} \\ \mathcal{T} & 0\end{array}\right)$. So $\left(\begin{array}{cc}0 & B \\ B & 0\end{array}\right)$ is isometric to the paired form.

(ii) $\pi \in\{x, x+1\}, n+v$ is odd and $U^{T} B U=\left(\begin{array}{cc}\mathcal{T} \mathcal{N}^{v}\left(I_{m n}+\mathcal{N}\right) & \mathcal{T} \\ \mathcal{T} & 0\end{array}\right)$.

(iii) $\pi \notin\{x, x+1\}, n+v$ is even and $U^{T} B U=\left(\begin{array}{cc}\mathcal{T} \mathcal{N}^{v}\left(\mathcal{P}+\mathcal{P}^{2}\right) & \mathcal{T} \\ \mathcal{T} & 0\end{array}\right)$.

If (i), (ii) or (ii) holds, let $D \in \operatorname{Diag}_{m n}(k)$ be the diagonal of $B$. Then the corresponding $G$-invariant quadratic forms are $\left\{\Delta\left(\begin{array}{cc}D & B \\ 0 & E\end{array}\right) \mid E \in \operatorname{Diag}_{m n}(k)\right\}$.

In case (i) $D=0$ and $\Delta\left(\begin{array}{ll}0 & B \\ 0 & E\end{array}\right)$ is isometric to $\Delta\left(\begin{array}{ll}0 & B \\ 0 & 0\end{array}\right)$.

In cases (ii) and (iii) we can choose $t$ so that $\left(B^{-1}\right)_{t, t} \neq 0$. Then $\Delta\left(\begin{array}{cc}D & B \\ 0 & E\end{array}\right)$ is isometric to $\Delta\left(\begin{array}{cc}D & B \\ 0 & \tau E_{t}\end{array}\right)$ for a unique $\tau \in\left(B^{-1}\right)_{t, t}^{-1} \mathcal{P}(k)$.

Proof. First note that $g_{2}=\left(\begin{array}{cc}I_{2 m n} & I_{2 m n} \\ 0 & I_{2 m n}\end{array}\right)$ and $g_{3}=\left(\begin{array}{cc}I_{2 m n} & \mathcal{P}_{2} \\ 0 & I_{2 m n}\end{array}\right)$, with respect to the basis $\mathcal{C}$ of $C_{n}(\pi)^{2}$. Here $\mathcal{P}_{2}$ denotes $\left(\begin{array}{cc}\mathcal{P} & 0 \\ 0 & \mathcal{P}\end{array}\right)$ in $\operatorname{Mat}_{2}(\mathrm{C}(\mathcal{P}))$.

Let $Q=\Delta\left(\begin{array}{ll}D & B \\ 0 & E\end{array}\right)$ be a quadratic form which polarizes to $\left(\begin{array}{cc}0 & B \\ B & 0\end{array}\right)$ and with $D, E \in$ $\operatorname{Diag}_{m n}(k)$. Then $Q$ is $G$-invariant if and only if $Q-g_{j}^{T} Q g_{j}$ is alternating, for $j=2,3$. One checks quickly that

$$
Q-g_{2}^{T} Q g_{2}=\left(\begin{array}{cc}
0 & D \\
D & D+B
\end{array}\right), \quad Q-g_{3}^{T} Q g_{3}=\left(\begin{array}{cc}
0 & D \mathcal{P}_{2} \\
\mathcal{P}_{2}^{T} D & \mathcal{P}_{2}^{T} D \mathcal{P}_{2}+\mathcal{P}_{2}^{T} B
\end{array}\right)
$$


Write $B=\left(\begin{array}{cc}\widetilde{B}_{1} & \widetilde{B}_{2} \\ \widetilde{B}_{2} & \widetilde{B}_{4}\end{array}\right)$, where $\widetilde{B}_{1}, \widetilde{B}_{2}, \widetilde{B}_{4} \in \mathrm{T}(\mathcal{P})$. Then $\widetilde{B}_{i}^{T}=\widetilde{B}_{i}$, and $\mathcal{P}^{T} \widetilde{B}_{i}=\widetilde{B}_{i} \mathcal{P}$, for $i=1,2,4$. So $D+B$ and $\mathcal{P}_{2}^{T} D \mathcal{P}_{2}+\mathcal{P}_{2}^{T} B$ are symmetric. Now $D+B$ is hollow if and only if $\mathcal{P}_{2}^{T}(D+B) \mathcal{P}_{2}$ is hollow. It follows that $D+B$ and $\mathcal{P}_{2}^{T} D \mathcal{P}_{2}+\mathcal{P}_{2}^{T} B$ are hollow if and only if $D+B$ and $\mathcal{P}_{2}^{T} B \mathcal{P}_{2}+\mathcal{P}_{2}^{T} B$ are hollow.

Now $\mathcal{P}_{2}^{T} B \mathcal{P}_{2}+\mathcal{P}_{2}^{T} B=\left(\begin{array}{cc}\widetilde{B}_{1}\left(\mathcal{P}+\mathcal{P}^{2}\right) & \widetilde{B}_{2}\left(\mathcal{P}+\mathcal{P}^{2}\right) \\ \widetilde{B}_{2}\left(\mathcal{P}+\mathcal{P}^{2}\right) & \widetilde{B}_{4}\left(\mathcal{P}+\mathcal{P}^{2}\right)\end{array}\right)$. We conclude that $Q$ is $G$-invariant if and only if each of $D+B, \widetilde{B}_{1}\left(\mathcal{P}+\mathcal{P}^{2}\right)$ and $\widetilde{B}_{4}\left(\mathcal{P}+\mathcal{P}^{2}\right)$ are hollow.

Notice that if $D+B$ is hollow if and only if $D$ is the diagonal of $B$. Next, we may assume that $\widetilde{B}_{1}=\mathcal{T} \mathcal{N}^{v}\left(I+\mathcal{N} f^{2}\right)$ and $\widetilde{B}_{4}=\mathcal{T} \mathcal{N}^{w}$, where $0<v<w \leq n+1, w-v$ is odd and $f \in K[\mathcal{N}]$.

If $v=n$, then $w=n+1$ and so $\widetilde{B}_{1}=\widetilde{B}_{4}=0$. This is case (i).

Suppose then that $v<n$. First let $\pi \in\{x, x+1\}$. Then $\mathcal{P}+\mathcal{P}^{2}=\mathcal{N}+\mathcal{N}^{2}$. So

$$
\widetilde{B}_{1}\left(\mathcal{P}+\mathcal{P}^{2}\right)=\mathcal{T}\left(\mathcal{N}^{v+1}\left(I_{m n}+\mathcal{N}^{2} f^{2}\right)+\mathcal{N}^{v+2}\left(I_{m n}+f^{2}\right)\right)
$$

Then Corollary 3.7 implies that $\widetilde{B}_{1}\left(\mathcal{P}+\mathcal{P}^{2}\right)$ is hollow if and only if $n+v$ is odd and $f=I_{m n}$ i.e. $\widetilde{B}_{1}=\mathcal{T} \mathcal{N}^{v}\left(I_{m n}+\mathcal{N}\right)$.

Next suppose $\mathcal{N}^{w} \neq 0$. Then Corollary 3.7 implies that $\widetilde{B}_{4}\left(\mathcal{P}+\mathcal{P}^{2}\right)=\mathcal{T}\left(\mathcal{N}^{w+1}+\right.$ $\mathcal{N}^{w+2}$ ) is hollow if and only if $w=n-1$. But then $w+n$ is odd. As $w-v$ is odd, we conclude that $\widetilde{B}_{4}\left(\mathcal{P}+\mathcal{P}^{2}\right)$ is hollow if and only if $\widetilde{B}_{4}=0$. This gives case (ii).

Finally let $\pi \notin\{x, x+1\}$. Then $\gamma:=\mathcal{E}+\mathcal{E}^{2}$ is a non-zero element of $K$ and $\mathcal{P}+\mathcal{P}^{2}=$ $\gamma+\mathcal{N}+\mathcal{N}^{2}$ in $K[\mathcal{N}]$. In this case

$$
\widetilde{B}_{1}\left(\mathcal{P}+\mathcal{P}^{2}\right)=\mathcal{T}\left(\mathcal{N}^{v}\left(\gamma+\mathcal{N}^{2}\left(I_{m n}+f^{2}\right)\right)+\mathcal{N}^{v+1}\left(I_{m n}+\left(\gamma+\mathcal{N}^{2}\right) f^{2}\right)\right)
$$

So by Corollary 3.7, $\widetilde{B}_{1}\left(\mathcal{P}+\mathcal{P}^{2}\right)$ is hollow if and only if $n+v$ is even and $f^{2}=\left(\gamma+\mathcal{N}^{2}\right)^{-1}$ i.e.

$$
\widetilde{B}_{1} f^{-2}=\mathcal{T} \mathcal{N}^{v}\left(\gamma+\mathcal{N}+\mathcal{N}^{2}\right)=\mathcal{T} \mathcal{N}^{v}\left(\mathcal{P}+\mathcal{P}^{2}\right)
$$

Now just as in the case $\pi \in\{x, x+1\}$ we have that $\widetilde{B}_{4}\left(\mathcal{P}+\mathcal{P}^{2}\right)$ is hollow if and only if $\widetilde{B}_{4}=0$.

Now suppose that $\Delta\left(\begin{array}{cc}D & B \\ 0 & E\end{array}\right)$ is a $G$-invariant quadratic form. Note that in case (i) $B$ is hollow and thus $D=0$. Now Lemma 6.6(i) applies. In the cases (ii) and (iii) we see from Corollary 3.7 that $B$ is not hollow. For if $n+v$ is odd then $\mathcal{N}^{j}(1+\mathcal{N})$ is not a square, for $j=v, v-1$, and if $n+v$ is even, then $\mathcal{N}^{j}\left(\gamma+\mathcal{N}+\mathcal{N}^{2}\right)$ is not a square, for $j=v, v-1$.

Now $B^{-1}=B^{-1} B B^{-T}$ is not hollow. Choose $t \in\{1, \ldots, m n\}$ such that $\left(B^{-1}\right)_{t, t} \neq 0$ and set $\nu=\left(B^{-1}\right)_{t, t}$. Since $D+B$ is hollow, it follows that $B^{-1}(D+B) B^{-T}$ is hollow. So $\nu=\left(B^{-1} D B^{-T}\right)_{t, t}$. Then Lemma 6.6(ii) shows that $\Delta\left(\begin{array}{cc}D & B \\ 0 & E\end{array}\right)$ is isometric to $\Delta\left(\begin{array}{cc}D & B \\ 0 & \tau E_{t}\end{array}\right)$, for a unique $\tau \in \nu^{-1} \mathcal{P}(k)$. 


\section{The modules $C_{n}(\infty)$ and $C_{n}(\infty)^{2}$}

In [1] Conlon defines $C_{n}(\infty)$ as a $2 n$-dimensional $k G$-module with the basis $\mathcal{B}:=$ $\left(a_{1}, \ldots, a_{n}, b_{1}, \ldots, b_{n}\right)$ such that

$$
n_{2}=\left(\begin{array}{cc}
0 & T_{2} \\
0 & 0
\end{array}\right), \quad n_{3}=\left(\begin{array}{cc}
0 & I_{n} \\
0 & 0
\end{array}\right)
$$

Note that up to conjugation by $\operatorname{diag}\left(\widetilde{I}_{n}, \widetilde{I}_{n}\right)$ the action on $C_{n}(\infty)$ equals the action on $C_{n}(x)$ given by (14), but with the roles of $n_{2}$ and $n_{3}$ interchanged. Hence $C_{n}(\infty)$ and $C_{n}(x)$ have the same symplectic and quadratic forms and the same isometry classes. For $C_{n}(x)$ we have $K=k, \mathcal{P}=\mathcal{N}=T_{0}$ and $\mathcal{T}=\widetilde{I}_{n}=H_{n+1}$. Also $\widetilde{I}_{n} \mathcal{T} \mathcal{N}^{v} \widetilde{I}_{n}=\mathcal{N}^{v} \mathcal{T}=$ $H_{n+1+v}$, for all integers $v \geq 0$. The following results for $C_{n}(\infty)$ are with respect to the basis $\mathcal{B}$. Theorem 7.5 implies

\section{Theorem 8.1.}

$\operatorname{Isom}\left(C_{n}(\infty)\right)=\left\{\left(\begin{array}{cc}0 & H_{n+1}+\sum_{i=1}^{\left\lfloor\frac{n}{2}\right\rfloor} \lambda_{i} H_{n+2 i} \\ H_{n+1}+\sum_{i=1}^{\left\lfloor\frac{n}{2}\right\rfloor} \lambda_{i} H_{n+2 i} & 0\end{array}\right) \mid \lambda_{1}, \ldots \lambda_{\left\lfloor\frac{n}{2}\right\rfloor} \in k\right\}$

Corollary 8.2. If $q=|k|$ then $\left|\operatorname{Isom}\left(C_{n}(\pi)\right)\right|=q^{\left\lfloor\frac{n}{2}\right\rfloor}$.

For quadratic forms, Theorem 7.7 gives:

Theorem 8.3. A bilinear form on $C_{n}(\infty)$ is the polarization of a G-invariant quadratic form if and only if $n$ is odd and the form is isometric to $\left(\begin{array}{cc}0 & H_{n+1}+H_{n+2} \\ H_{n+1}+H_{n+2} & 0\end{array}\right)$.

In this case the G-invariant quadratic forms which polarize to the above symplectic form are $\Delta\left(\begin{array}{cc}E_{\frac{n+1}{2}} & H_{n+1}+H_{n+2} \\ 0 & D\end{array}\right)$ for $D \in \operatorname{Diag}_{n}(k)$. Each of these is isometric to $\Delta\left(\begin{array}{cc}E_{\frac{n+1}{2}} & H_{n+1}+H_{n+2} \\ 0 & \mu E_{\frac{n+1}{2}}\end{array}\right)$ for a unique $\mu \in \mathcal{P}(k)$.

The remaining three statements are with respect to the ordered basis

$$
\mathcal{C}:=\left(\left(a_{1}, 0\right), \ldots,\left(a_{n}, 0\right),\left(0, a_{1}\right), \ldots,\left(0, a_{n}\right),\left(b_{1}, 0\right), \ldots,\left(b_{n}, 0\right),\left(0, b_{1}\right), \ldots,\left(0, b_{n}\right)\right)
$$

on $C_{n}(\infty)^{2}$ and follow from Theorem 7.8, Corollary 7.9 and Theorem 7.10, respectively. 
Theorem 8.4. Each symplectic form on $C_{n}(\infty)^{2}$ is isometric to one form $\left(\begin{array}{cc}0 & B \\ B & 0\end{array}\right)$ with

$$
B=\left(\begin{array}{cc}
H_{v}+\sum_{i=1}^{\frac{w-v-1}{2}} \lambda_{i} H_{v-1+2 i} & H_{n+1}+\sum_{i=1}^{\left\lfloor\frac{2 n-w+2}{2}\right\rfloor} \mu_{i} H_{n+i} \\
H_{n+1}+\sum_{i=1}^{\left\lfloor\frac{2 n-w+2}{2}\right\rfloor} \mu_{i} H_{n+i} & H_{w}
\end{array}\right)
$$

Here $n+1<v<w \leq 2 n+1, w-v$ is odd, $\lambda_{i}, \mu_{i} \in k$ and $\mu_{1} \neq 1_{k}$

Corollary 8.5. If $k=\mathbb{F}_{q}$ then $\left|\operatorname{Isom}\left(C_{n}(\infty)^{2}\right)\right|= \begin{cases}n q^{\frac{n-2}{2}}, & \text { if } n \text { is even, } \\ \left(\frac{n+1}{2} q+\frac{n-1}{2}\right) q^{\frac{n-3}{2}}, & \text { if } n \text { is odd. }\end{cases}$

Theorem 8.6. There is a G-invariant quadratic form on $C_{n}(\infty)^{2}$ which polarizes to $\left(\begin{array}{cc}0 & B \\ B & 0\end{array}\right)$ if and only if there is a unit $U \in \operatorname{Mat}_{2}\left(\mathrm{C}\left(T_{2}\right)\right)$ so that one of the following holds:

(i) $U^{T} B U=\left(\begin{array}{cc}0 & H_{n+1} \\ H_{n+1} & 0\end{array}\right)$.

(ii) $U^{T} B U=\left(\begin{array}{cc}H_{v}+H_{v+1} & H_{n+1} \\ H_{n+1} & 0\end{array}\right)$, where $v>n+1$ and $v$ is even.

If (i) holds, $\left\{\Delta\left(\begin{array}{cc}0 & B \\ 0 & D\end{array}\right) \mid D \in \operatorname{Diag}_{n}(k)\right\}$ are the corresponding $G$-invariant quadratic forms. Each of these are isometric to $\Delta\left(\begin{array}{cc}0 & B \\ 0 & 0\end{array}\right)$.

If (ii) holds, choose $t$ so that $\left(B^{-1}\right)_{t, t} \neq 0$. Then $\Delta\left(\begin{array}{cc}D & B \\ 0 & E\end{array}\right)$ is isometric to $\Delta\left(\begin{array}{cc}D & B \\ 0 & \tau E_{t}\end{array}\right)$, for a unique $\tau \in\left(B^{-1}\right)_{t, t}^{-1} \mathcal{P}(k)$.

\section{References}

[1] S.B. Conlon, Modular representations of $C_{2} \times C_{2}$, J. Aust. Math. Soc. 10 (1969) 363-366.

[2] R. Gow, W. Willems, A note on green correspondence and forms, Comm. Algebra 23 (4) (1995) 1239-1248.

[3] R. Gow, W. Willems, Quadratic geometries, projective modules and idempotents, J. Algebra 160 (1993) 257-272.

[4] L.C. Grove, Classical Groups and Geometric Algebra, Graduate Studies in Mathematics, vol. 39, AMS, 2001.

[5] O. Taussky, H. Zassenhaus, On the similarity transformation between a matrix and its transpose, Pacific J. Math. 9 (1959) 893-896.

[6] G.E. Wall, On the conjugacy classes in the unitary, symplectic and orthogonal groups, J. Aust. Math. Soc. 3 (1963) 1-62. 